\title{
The exponentiated half-logistic odd lindley-G family of dis- tributions with applications
}

\author{
Whatmore Sengweni*, Brodrick Oluyede, Boikanyo Makubate \\ Department of Mathematical Statistics, Botswana International University of Science and Technology, P. Bag 16, Palapye, Botswana.
}

\begin{abstract}
A new generalized family of models called the Exponentiated Half Logistic Odd Lindley-G (EHLOL-G) distribution is developed and presented. Some explicit expressions for the structural properties including moments, conditional moments, mean and median deviations, distribution of the order statistics, probability weighted moments and Rényi entropy are derived. We applied the maximum likelihood estimation technique to estimate the parameters of the model and a simulation study is conducted to examine the efficiency of the maximum likelihood estimators. The special case of the EHLOL-Weibull (EHLOL-W) distribution is fitted to two real data sets.
\end{abstract}

Keywords: Generalized-G distribution, exponentiated distribution, half logistic distribution, odd-lindley distribution, maximum likelihood estimation.

2020 MSC: 62E99, 60E05.

(C)2021 All rights reserved.

\section{Introduction}

The density function of the Half Logistic (HL) distribution does not possess many desirable properties for data modeling ([2]). The fact that most real data exhibits non-monotonic hazard rate function shapes act as a limitation to the applicability of the HL distribution in data modeling. Recently, some researchers have generalized the HL distribution and this include work by $[2,6,7,9]$.

In this note, we propose a new flexible family of distributions called the Exponentiated Half Logistic Odd Lindley-G (EHLOL-G) distribution using the exponentiated HL distribution as the generator and study its mathematical properties. By so doing, we will improve the flexibility of the exponentiated HL distribution and the baseline distribution.

The results in this note are organized in the following manner. Section 2 contain the EHLOL-G family of distributions and its sub-models, hazard rate function, quantile function, series expansion of the density function and some special cases of the EHLOL-G family of distributions. In Section 3, moments, generating function and incomplete moments are given. Section 4 contains results on the distribution of

\footnotetext{
*Corresponding author

Email addresses: whatiegumbo@gmail.com (Whatmore Sengweni), oluyedeo@biust.ac.bw (Brodrick Oluyede),

makubateb@biust.ac.bw (Boikanyo Makubate)
}

doi: $10.22436 /$ jnsa.014.05.01

Received: 2020-10-28 Revised: 2020-11-23 Accepted: 2020-12-02 
order statistics, probability weighted moments and Rényi entropy. Presented in Section 5 is the the estimation of the parameters of the EHLOL-G family of distributions via the method of maximum likelihood, followed by a Monte Carlo simulation study to examine the bias and mean square error of the maximum likelihood estimators in Section 6. Some applications to real data sets are given in Section 7, followed by some concluding remarks in Section 8.

\section{The exponentiated half-logistic odd lindley-G distribution}

In this section, we derive some of the statistical properties of the Exponentiated Half-Logistic Odd Lindley-G (EHLOL-G) family of distributions including special cases, expansion of the density, hazard rate function and quantile function.

\subsection{The model}

The Odd-Lindley-G family of distributions was developed by [10] and its cumulative distribution function (cdf) is given by

$$
\mathrm{F}_{\mathrm{OL}-\mathrm{G}}(x ; \lambda, \underline{\zeta})=1-\frac{\lambda+\overline{\mathrm{G}}(x ; \underline{\zeta})}{(1+\lambda) \overline{\mathrm{G}}(x ; \underline{\zeta})} \exp \left[-\lambda \frac{\mathrm{G}(x ; \underline{\zeta})}{\overline{\mathrm{G}}(x ; \underline{\zeta})}\right]
$$

where $\mathrm{G}(\mathrm{x} ; \underline{\zeta})$ is the baseline cdf. [6] developed the type I half-logistic (TIHL) family of distributions with the cdf

$$
F(x ; \lambda, \underline{\zeta})=\frac{1-(1-G(x ; \underline{\zeta}))^{\lambda}}{1+(1-G(x ; \underline{\zeta}))^{\lambda}}
$$

where $\lambda>0$, is the shape parameter. If $\lambda=1$, we have

$$
F(x ; \underline{\zeta})=\frac{G(x ; \underline{\zeta})}{1+\bar{G}(x ; \underline{\zeta})}, \text { where } \bar{G}(x ; \underline{\zeta})=1-\mathrm{G}(x ; \underline{\zeta}) .
$$

The cdf and pdf of the new exponentiated half-logistic Odd Lindley-G (EHLOL-G) family of distributions are, respectively given by

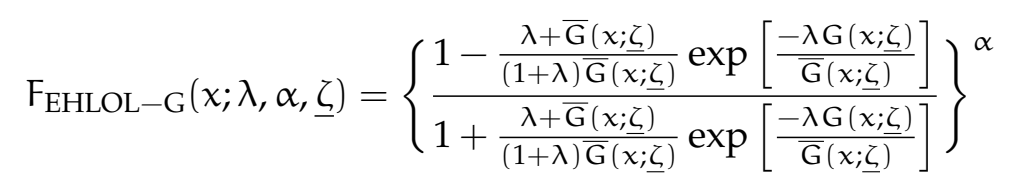

and

$$
f_{\text {EHLOL-G }}(x ; \lambda, \alpha, \underline{\zeta})=2 \alpha \lambda^{2} g(x ; \underline{\zeta}) \frac{\exp \left[\frac{-\lambda G(x ; \underline{\zeta})}{\bar{G}(x ; \underline{\zeta})}\right]\left\{1-\frac{\lambda+\bar{G}(x ; \zeta)}{(1+\lambda) \bar{G}(x ; \underline{\zeta})} \exp \left[\frac{-\lambda G(x ; \zeta)}{\bar{G}(x ; \underline{\zeta})}\right]\right\}^{\alpha-1}}{(1+\lambda) \bar{G}^{3}(x ; \underline{\zeta})\left\{1+\frac{\lambda+\bar{G}(x ; \underline{\zeta})}{(1+\lambda) \bar{G}(x ; \underline{\zeta})} \exp \left[\frac{-\lambda G(x ; \zeta)}{\bar{G}(x ; \underline{\zeta})}\right]\right\}^{\alpha+1}}
$$

where $g(x ; \underline{\zeta})=\frac{\mathrm{dG}(x ; \underline{\zeta})}{\mathrm{d} x}, \lambda>0, \alpha>0$ and $\underline{\zeta}$ is the vector of parameters for the baseline cdf $\mathrm{G}$. If a random variable $X$ has the EHLOL-G family of distributions, we write $X \sim E H L O L-G(\lambda, \alpha, \underline{\zeta})$.

\subsection{Hazard rate and quantile functions}

The hazard rate and quantile functions of the EHLOL-G family of distributions are presented in this 
section. The hazard rate function (hrf) is given by

$$
\begin{aligned}
h(x)= & 2 \alpha \lambda^{2} g(x ; \underline{\zeta}) \frac{\exp \left[\frac{-\lambda G(x ; \zeta)}{\bar{G}(x ; \underline{\zeta})}\right]\left\{1-\frac{\lambda+\bar{G}(x ; \zeta)}{(1+\lambda) \bar{G}(x ; \underline{\zeta})} \exp \left[\frac{-\lambda G(x ; \underline{\zeta})}{\bar{G}(x ; \underline{\zeta})}\right]\right\}^{\alpha-1}}{(1+\lambda) \bar{G}^{3}(x ; \underline{\zeta})\left\{1+\frac{\lambda+\bar{G}(x ; \underline{\zeta})}{(1+\lambda) \bar{G}(x ; \zeta)} \exp \left[\frac{-\lambda G(x ; \underline{\zeta})}{\bar{G}(x ; \underline{\zeta})}\right]\right\}^{\alpha+1}} \\
& \times\left[1-\left\{\frac{1-\frac{\lambda+\bar{G}(x ; \underline{\zeta})}{(1+\lambda) \bar{G}(x ; \underline{\zeta})} \exp \left[\frac{-\lambda G(x ; \zeta)}{\bar{G}(x ; \underline{\zeta})}\right]}{1+\frac{\lambda+\bar{G}(x ; \underline{\zeta})}{(1+\lambda) \bar{G}(x ; \underline{\zeta})} \exp \left[\frac{-\lambda G(x ; \zeta)}{\bar{G}(x ; \underline{\zeta})}\right]}\right]^{-1} .\right.
\end{aligned}
$$

The quantile function $\mathrm{Q}(\mathrm{u})$ is obtained by solving the non-linear equation

$$
\mathrm{F}_{\mathrm{EHLOL}-\mathrm{G}}(\mathrm{Q}(\mathrm{u}))=\left\{\frac{1-\frac{\lambda+1-\mathrm{G}(\mathrm{Q}(\mathfrak{u}))}{(1+\lambda)(1-\mathrm{G}(\mathrm{Q}(\mathfrak{u})))} \exp \left[\frac{-\lambda \mathrm{G}(\mathrm{Q}(\mathfrak{u}))}{1-\mathrm{G}(\mathrm{Q}(\mathfrak{u}))}\right]}{1+\frac{\lambda+1-\mathrm{G}(\mathrm{Q}(\mathfrak{u}))}{(1+\lambda)(1-\mathrm{G}(\mathrm{Q}(\mathfrak{u})))} \exp \left[\frac{-\lambda \mathrm{G}(\mathrm{Q}(\mathfrak{u}))}{1-\mathrm{G}(\mathrm{Q}(\mathfrak{u}))}\right]}\right\}^{\alpha}=\mathfrak{u},
$$

$0<u<1$, so that,

$$
-\frac{\lambda+1-G(Q(u))}{(1+\lambda)(1-G(Q(u)))} \exp \left[-\lambda \frac{G(Q(u))}{1-G(Q(u))}\right]=\frac{u^{\frac{1}{\alpha}}-1}{u^{\frac{1}{\alpha}}+1} .
$$

By multiplying both sides of the above equation by $\exp [-(1+\lambda)]$ and $(1+\lambda)$, we obtain

$$
-\frac{\lambda+1-G(Q(u))}{1-G(Q(u))} \exp \left[-\frac{\lambda+1-G(Q(u))}{1-G(Q(u))}\right]=\frac{\left(u^{\frac{1}{\alpha}}-1\right)(1+\lambda)}{u^{\frac{1}{\alpha}}+1} \exp [-(1+\lambda)]
$$

It can be seen that $-\frac{\lambda+1-G(Q(u))}{1-G(Q(u))}$ is a Lambert $W$ function of the real argument $\frac{\left(\mathfrak{u}^{\frac{1}{\alpha}}-1\right)(1+\lambda)}{\mathfrak{u}^{\frac{1}{\alpha}}+1} \exp [-(1+\lambda)]$. The Lambert $W$ function is defined by

$$
W(x) e^{W(x)}=x
$$

which has 2 real branches with a branching point located at $\left(-e^{-1}, 1\right)$. The lower branch, $W_{-1}(x)$, is defined in the interval $\left[-e^{-1}, 1\right]$ and has a negative singularity for $x \rightarrow 0^{-}$. The upper branch, $W_{0}(x)$, is defined for $x \in\left[-e^{-1}, \infty\right]$. Thus, we have

$$
W\left[\frac{\left(u^{\frac{1}{\alpha}}-1\right)(1+\lambda)}{u^{\frac{1}{\alpha}}+1} \exp [-(1+\lambda)]=-\left[1+\frac{\lambda}{1-G(Q(u))}\right] .\right.
$$

For any $\lambda>0$ and $u \in(0,1)$, we have $\left[1+\frac{\lambda}{1-G(Q(u))}\right]>1$ and $\frac{\left(u^{\frac{1}{\alpha}}-1\right)(1+\lambda)}{u^{\frac{1}{\alpha}}+1} \exp [-(1+\lambda)]<0$, we can write equation (2.3) as

$$
W_{-1}\left[\frac{\left(u^{\frac{1}{\alpha}}-1\right)(1+\lambda)}{u^{\frac{1}{\alpha}}+1} \exp [-(1+\lambda)]\right]=-\left[1+\frac{\lambda}{1-G(Q(u))}\right]
$$

Thus, the quantile function is given by,

$$
\mathrm{Q}(\mathrm{u})=\mathrm{G}^{-1}\left\{1+\lambda\left[1+\mathrm{W}_{-1}\left[\frac{\left(\mathrm{u}^{\frac{1}{\alpha}}-1\right)(1+\lambda)}{\mathrm{u}^{\frac{1}{\alpha}}+1} \exp [-(1+\lambda)]\right]\right]^{-1}\right\}
$$




\subsection{Expansion of density}

Let

$$
Y=\left\{1-\frac{\lambda+\bar{G}(x ; \underline{\zeta})}{(1+\lambda) \bar{G}(x ; \underline{\zeta})} \exp \left[\frac{-\lambda G(x ; \underline{\zeta})}{\bar{G}(x ; \underline{\zeta})}\right]\right\}^{\alpha-1}
$$

and

$$
Z=\left\{1+\frac{\lambda+\bar{G}(x ; \underline{\zeta})}{(1+\lambda) \bar{G}(x ; \underline{\zeta})} \exp \left[\frac{-\lambda G(x ; \underline{\zeta})}{\bar{G}(x ; \underline{\zeta})}\right]\right\}^{-\alpha-1}
$$

By making use of the generalized binomial series expansion we obtained

$$
Y=\sum_{j=0}^{\infty}(-1)^{j}\left(\begin{array}{c}
\alpha-1 \\
j
\end{array}\right)\left[\frac{\lambda+\bar{G}(x ; \underline{\zeta})}{(1+\lambda) \bar{G}(x ; \underline{\zeta})}\right]^{j} \exp \left[\frac{-\lambda j G(x ; \underline{\zeta})}{\bar{G}(x ; \underline{\zeta})}\right],
$$

and

$$
Z=\sum_{i=0}^{\infty}\left(\begin{array}{c}
-\alpha-1 \\
i
\end{array}\right)\left[\frac{\lambda+\bar{G}(x ; \underline{\zeta})}{(1+\lambda) \bar{G}(x ; \underline{\zeta})}\right]^{i} \exp \left[\frac{-\lambda i G(x ; \underline{\zeta})}{\bar{G}(x ; \underline{\zeta})}\right]
$$

Putting together equations (2.4) and (2.5) in equation (2.2) we obtained

$$
f(x)=\frac{2 \alpha \lambda^{2} g(x ; \underline{\zeta})}{(1+\lambda) \bar{G}^{3}(x ; \underline{\zeta})} \sum_{j, i=0}^{\infty}(-1)^{j}\left(\begin{array}{c}
\alpha-1 \\
j
\end{array}\right)\left(\begin{array}{c}
-\alpha-1 \\
i
\end{array}\right)\left[\frac{\lambda+\bar{G}(x ; \underline{\zeta})}{(1+\lambda) \bar{G}(x ; \underline{\zeta})}\right]^{j+i} \exp \left[\frac{-\lambda(j+i+1) G(x ; \underline{\zeta})}{\bar{G}(x ; \underline{\zeta})}\right] .
$$

By applying the binomial expansion

$$
(\lambda+\bar{G}(x ; \underline{\zeta}))^{j+i}=\sum_{q=0}^{j+i}\left(\begin{array}{c}
j+i \\
q
\end{array}\right) \lambda^{j+i} \bar{G}(x ; \underline{\zeta})^{j+i-q},
$$

and the exponential series expansion, one can write the EHLOG-G pdf as

$$
\begin{aligned}
f(x)= & 2 \alpha g(x ; \underline{\zeta}) \sum_{j, i, k=0}^{\infty} \sum_{q=0}^{j+i} \frac{(-1)^{j+k} \lambda^{j+i+k+2}(j+i+1)^{k}}{k !(1+\lambda)^{j+i+1}}\left(\begin{array}{c}
\alpha-1 \\
j
\end{array}\right)\left(\begin{array}{c}
-\alpha-1 \\
i
\end{array}\right) \\
& \times\left(\begin{array}{c}
j+i \\
q
\end{array}\right) G(x ; \underline{\zeta})^{k} \bar{G}(x ; \underline{\zeta})^{-(k+q+3)} .
\end{aligned}
$$

We apply the following generalized binomial series expansion

$$
(1-z)^{-p}=\sum_{l=0}^{\infty}(-1)^{l}\left(\begin{array}{c}
-p \\
l
\end{array}\right) z^{l} \text { for }|z|<1 \text { and } p>0,
$$

to express equation (2.6) as

$$
\begin{aligned}
f(x)= & 2 \alpha g(x ; \underline{\zeta}) \sum_{j, i, k, l=0}^{\infty} \sum_{q=0}^{j+i} \frac{(-1)^{j+k+l} \lambda^{j+i+k+2}(j+i+1)^{k}}{k !(1+\lambda)^{j+i+1}}\left(\begin{array}{c}
\alpha-1 \\
j
\end{array}\right)\left(\begin{array}{c}
-\alpha-1 \\
i
\end{array}\right) \\
& \times\left(\begin{array}{c}
j+i \\
q
\end{array}\right)\left(\begin{array}{c}
-(k+q+3) \\
l
\end{array}\right) G(x ; \underline{\zeta})^{k+l} .
\end{aligned}
$$


Consequently, we can write

$$
f(x)=\sum_{k, l=0}^{\infty} a_{k, l} h_{k+l+1}(x)
$$

where

$$
a_{k, l}=2 \alpha \sum_{j, i=0}^{\infty} \sum_{q=0}^{j+i} \frac{(-1)^{j+k+l} \lambda^{j+i+k+2}(j+i+1)^{k}}{k !(1+\lambda)^{j+i+1}(k+l+1)}\left(\begin{array}{c}
\alpha-1 \\
j
\end{array}\right)\left(\begin{array}{c}
-\alpha-1 \\
i
\end{array}\right)\left(\begin{array}{c}
j+i \\
q
\end{array}\right)\left(\begin{array}{c}
-(k+q+3) \\
l
\end{array}\right),
$$

and $h_{k+l+1}(x)=(k+l+1) g(x ; \zeta) G(x, \underline{\zeta})^{k+l}$ is the Exp-G density with power parameter $(k+l+1)$. Other several mathematical properties of the EHLOL-G family can be obtained from the properties of the Exp-G family.

\subsection{Some sub-families of EHLOL-G family of distributions}

Here we consider some sub-families of the EHLOL-G family of distributions.

- If $\alpha=1$, we obtain a new Half Logistic Odd Lindley-G family of distributions with pdf

$$
f_{H L O L-G}(x)=\frac{2 \lambda^{2} g(x ; \xi) \exp \left[\frac{-\lambda G(x ; \underline{\zeta})}{\bar{G}(x ; \underline{\zeta})}\right]}{(1+\lambda) \bar{G}^{3}(x ; \underline{\zeta})\left\{1+\frac{\lambda+\bar{G}(x ; \underline{\zeta})}{(1+\lambda) \bar{G}(x ; \underline{\zeta})} \exp \left[\frac{-\lambda G(x ; \underline{\zeta})}{\bar{G}(x ; \underline{\zeta})}\right]\right\}^{2}} .
$$

- If $\lambda=1$, we obtain a new family of distributions with the pdf

$$
f_{\mathrm{OEHL}-\mathrm{G}}(x)=\frac{\alpha g(x ; \underline{\zeta}) \exp \left[\frac{-\mathrm{G}(x ; \underline{\zeta})}{\overline{\mathrm{G}}(x ; \underline{\zeta})}\right]\left\{1-\frac{\mathrm{G}(x ; \underline{\zeta})}{2 \overline{\mathrm{G}}(x ; \underline{\zeta})} \exp \left[\frac{-\mathrm{G}(x ; \underline{\zeta})}{\overline{\mathrm{G}}(x ; \underline{\zeta})}\right]\right\}^{\alpha-1}}{\overline{\mathrm{G}}^{3}(x ; \underline{\zeta})\left\{1+\frac{\mathrm{G}(x ; \underline{\zeta})}{2 \overline{\mathrm{G}}(x ; \underline{\zeta})} \exp \left[\frac{-\mathrm{G}(x ; \underline{\zeta})}{\overline{\mathrm{G}}(\mathrm{x} ; \underline{\zeta})}\right]\right\}^{\alpha+1}} .
$$

- If $\alpha=1$ and $\lambda=1$, we obtain a new family of distributions with pdf

$$
f(x)=\frac{g(x ; \underline{\zeta}) \exp \left[\frac{-G(x ; \bar{\zeta})}{\bar{G}(x ; \underline{\zeta})}\right]}{\bar{G}^{3}(x ; \underline{\zeta})\left\{1+\frac{1+\bar{G}(x ; \underline{\zeta})}{2 \bar{G}(x ; \underline{\zeta})} \exp \left[\frac{-G(x ; \underline{\zeta})}{\bar{G}(x ; \underline{\zeta})}\right]\right\}^{2}} .
$$

\subsection{Some special cases}

Some special cases of the EHLOL-G family of distributions are presented in this section, cases when the baseline distribution function $\mathrm{G}(\mathrm{x} ; \zeta)$ are Weibull, Uniform, Kumaraswamy and Fréchet distributions, respectively. Plots of the pdf, and hrf are also presented.

\subsubsection{EHLOL-Weibull distribution}

Suppose the baseline distribution is the Weibull distribution with cdf and pdf given by $G(x ; a, b)=$ $1-e^{-(x / b)^{a}}$ and $g(x ; a, b)=a b^{-a} x^{a-1} e^{-(x / b)^{a}}$, for $a, b>0$ and $x>0$, then, the EHLOL-Weibull (EHLOL-W) distribution has cdf and pdf given by

$$
F_{\text {EHLOL }-W}(x)=\left\{\frac{1-\frac{\lambda+e^{-(x / b)^{a}}}{(1+\lambda) e^{-(x / b)^{a}}} \exp \left[\frac{-\lambda\left(1-e^{-(x / b)^{a}}\right)}{e^{-(x / b)^{a}}}\right]}{1+\frac{\lambda+e^{-(x / b)^{a}}}{(1+\lambda) e^{-(x / b)^{a}}} \exp \left[\frac{-\lambda\left(1-e^{-(x / b)^{a}}\right)}{e^{-(x / b)^{a}}}\right]}\right\}^{\alpha}
$$


and

$$
\begin{aligned}
f_{\text {EHLOL }-W}(x)= & 2 \alpha \lambda^{2} a b^{-a} x^{a-1} e^{-(x / b)^{a}} \\
& \times \frac{\exp \left[\frac{-\lambda\left(1-e^{-(x / b)^{a}}\right)}{e^{-(x / b)^{a}}}\right]\left\{1-\frac{\lambda+e^{-(x / b)^{a}}}{(1+\lambda) e^{-(x / b)^{a}}} \exp \left[\frac{-\lambda\left(1-e^{-(x / b)^{a}}\right)}{e^{-(x / b)^{a}}}\right]\right\}^{\alpha-1}}{(1+\lambda)\left(e^{-(x / b)^{a}}\right)^{3}\left\{1+\frac{\lambda+e^{-(x / b)}}{(1+\lambda) e^{-(x / b) a}} \exp \left[\frac{-\lambda\left(1-e^{-(x / b)^{a}}\right)}{e^{-(x / b)^{a}}}\right]\right\}^{\alpha+1}},
\end{aligned}
$$

respectively.
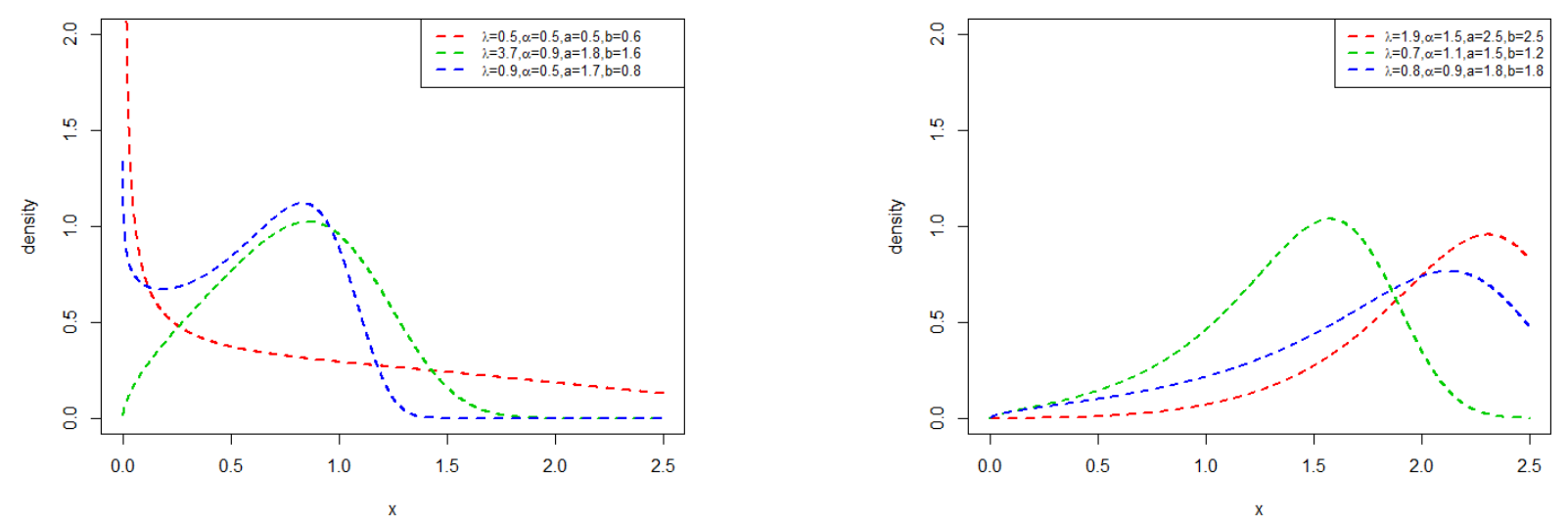

Figure 1: Plots of EHLOL-W density function.

Figures 1 and 2 show the plots of the density function and the hazard rate function of the EHLOL$\mathrm{W}$ distribution, respectively. The pdf of the EHLOL-W distribution can take various shapes including the reverse J, uni-modal, almost symmetric, left skewed and right skewed. The plots of the hrf of the EHLOL-W distribution exhibits decreasing, increasing and bathtub shapes.
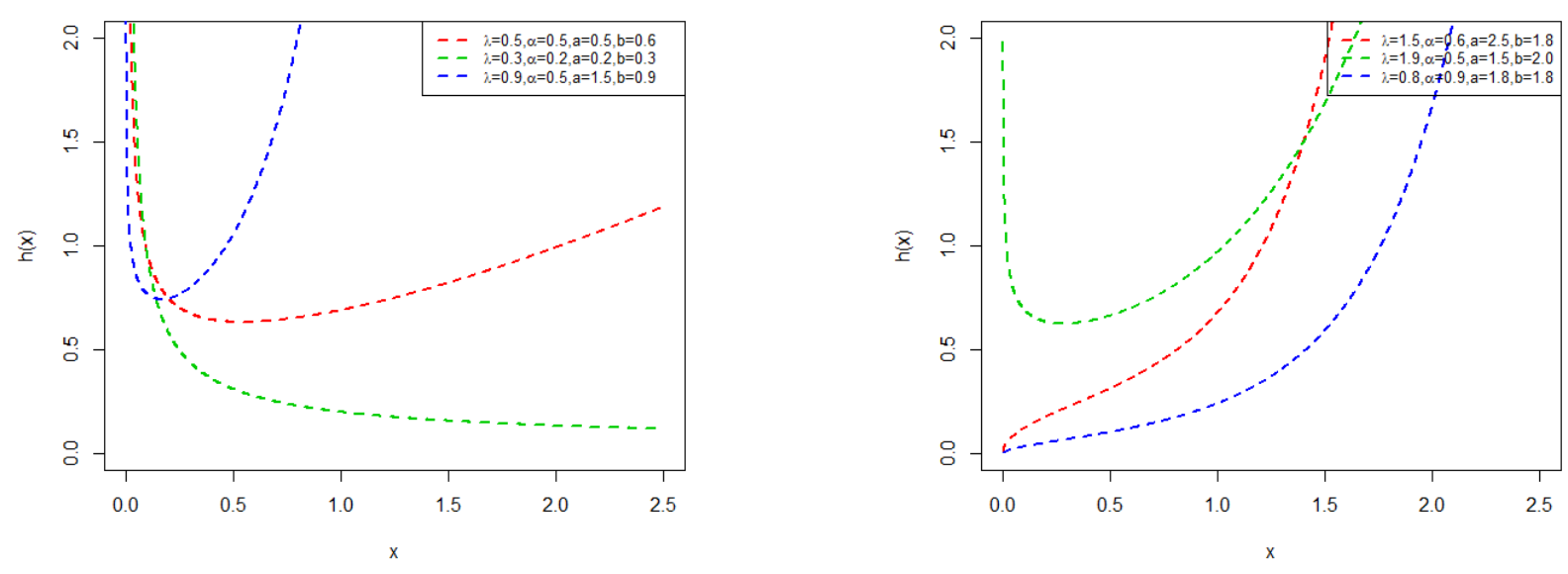

Figure 2: Plots of EHLOL-W hazard rate function.

\subsubsection{EHLOL-Uniform distribution}

Let the Uniform distribution with cdf and pdf given by $G(x ; \theta)=\frac{\chi}{\theta}$ and $g(x ; \theta)=\frac{1}{\theta}$ for $0<x<\theta<\infty$ be the baseline distribution. Then, the EHLOL-Uniform (EHLOL-U) distribution has cdf and pdf given 
by

$$
\mathrm{F}_{\text {EHLOL }-\mathrm{U}}(\mathrm{x})=\left\{\frac{1-\frac{\lambda+\left(1-\frac{\chi}{\theta}\right)}{(1+\lambda)\left(1-\frac{\chi}{\theta}\right)} \exp \left[\frac{-\lambda x}{\theta\left(1-\frac{x}{\theta}\right)}\right]}{1+\frac{\lambda+\left(1-\frac{x}{\theta}\right)}{(1+\lambda)\left(1-\frac{x}{\theta}\right)} \exp \left[\frac{-\lambda x}{\theta\left(1-\frac{x}{\theta}\right)}\right]}\right\}^{\alpha}
$$

and

$$
f_{\text {EHLOL }-\mathrm{u}}(\mathrm{x})=\frac{2 \alpha \lambda^{2}}{\theta} \frac{\exp \left[\frac{-\lambda x}{\theta\left(1-\frac{x}{\theta}\right)}\right]\left\{1-\frac{\lambda+\left(1-\frac{\chi}{\theta}\right)}{(1+\lambda)\left(1-\frac{x}{\theta}\right)} \exp \left[\frac{-\lambda x}{\theta\left(1-\frac{x}{\theta}\right)}\right]\right\}^{\alpha-1}}{(1+\lambda)\left(1-\frac{x}{\theta}\right)^{3}\left\{1+\frac{\lambda+\left(1-\frac{\chi}{\theta}\right)}{(1+\lambda)\left(1-\frac{x}{\theta}\right)} \exp \left[\frac{-\lambda x}{\theta\left(1-\frac{x}{\theta}\right)}\right]\right\}^{\alpha+1}}
$$
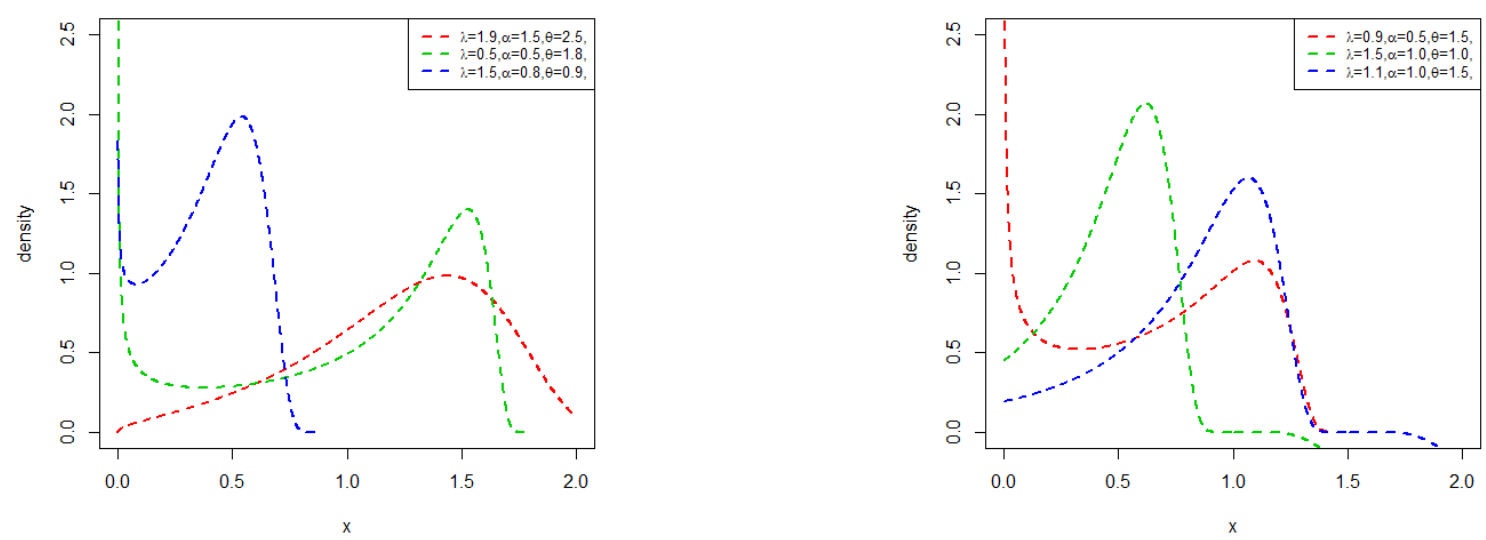

Figure 3: Plots of EHLOL-U density function.

Figures 3 and 4 show the plots of the pdf and hrf of the EHLOL-U distribution for different parameter values. The plots revealed that the pdf can take various shapes including the uni-modal, left skewed and right skewed. The hrf of the EHLOL-U distribution has increasing and bathtub shapes.
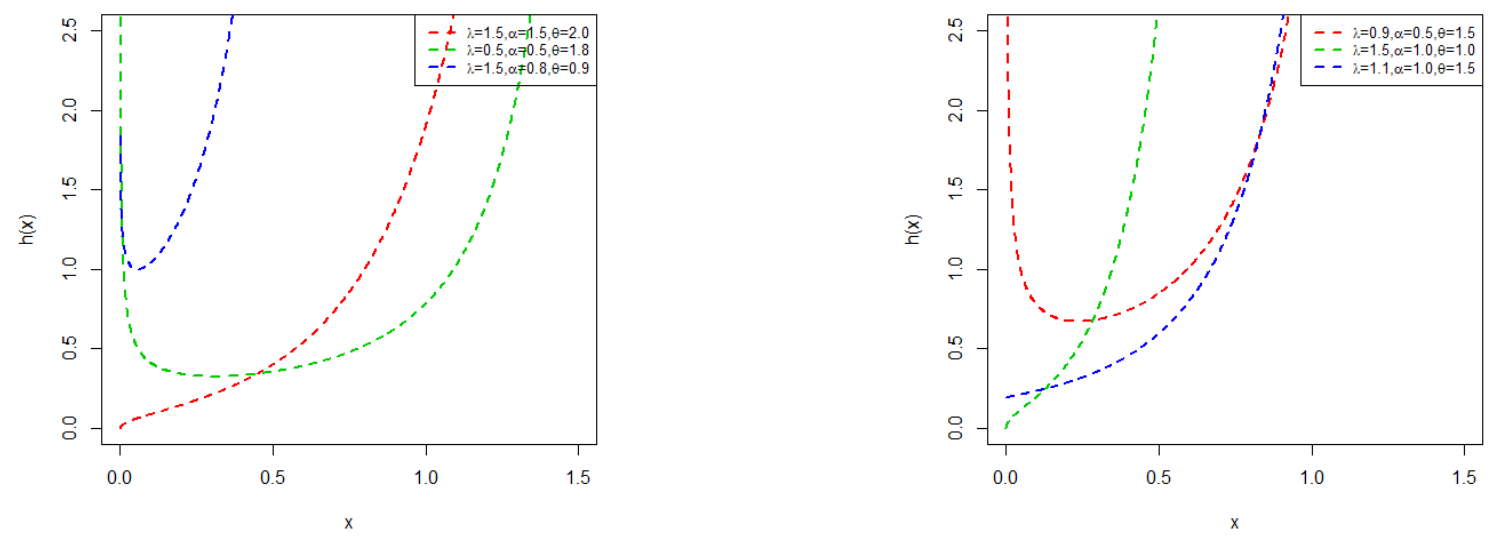

Figure 4: Plots of EHLOL-U hazard rate function.

\subsubsection{EHLOL-Kumaraswamy distribution}

Suppose the cdf and pdf of the baseline distribution are given by $G(x ; a, b)=1-\left(1-x^{a}\right)^{b}$ and 
$g(x ; a, b)=a b x^{a-1}\left(1-x^{a}\right)^{b-1}$ for $a, b>0$ and $x>0$. Then, the EHLOL-Kumaraswamy (EHLOL-Kum) distribution has cdf and pdf given by

$$
\text { FEHLOL-Kum }(x)=\left\{\frac{1-\frac{\lambda+\left(1-\chi^{a}\right)^{b}}{(1+\lambda)\left(1-\chi^{a}\right)^{b}} \exp \left[\frac{-\lambda\left(1-\left(1-\chi^{a}\right)^{b}\right)}{\left(1-\chi^{a}\right)^{b}}\right]}{1+\frac{\lambda+\left(1-\chi^{a}\right)^{b}}{(1+\lambda)\left(1-\chi^{a}\right)^{b}} \exp \left[\frac{-\lambda\left(1-\left(1-\chi^{a}\right)^{b}\right)}{\left(1-\chi^{a}\right)^{b}}\right]}\right\}^{\alpha}
$$

and

$$
\begin{aligned}
f_{\text {EHLOL-Kum }}(x)= & 2 \alpha \lambda^{2} a b x^{a-1}\left(1-x^{a}\right)^{b-1} \\
& \times \frac{\exp \left[\frac{-\lambda\left(1-\left(1-\chi^{a}\right)^{b}\right)}{\left(1-\chi^{a}\right)^{b}}\right]\left\{1-\frac{\lambda+\left(1-x^{a}\right)^{b}}{(1+\lambda)\left(1-x^{a}\right)^{b}} \exp \left[\frac{-\lambda\left(1-\left(1-\chi^{a}\right)^{b}\right)}{\left(1-\chi^{a}\right)^{b}}\right]\right\}^{\alpha-1}}{(1+\lambda)\left(1-\chi^{a}\right)^{3 b}\left\{1+\frac{\lambda+\left(1-\chi^{a}\right)^{b}}{(1+\lambda)\left(1-x^{a}\right)^{b}} \exp \left[\frac{-\lambda\left(1-\left(1-\chi^{a}\right)^{b}\right)}{\left(1-\chi^{a}\right)^{b}}\right]\right\}^{\alpha+1}} .
\end{aligned}
$$

Figure 5 shows the plots of the EHLOL-Kum distribution for different parameter values. The pdf can take various shapes including reverse J, uni-modal, left skewed and right skewed.
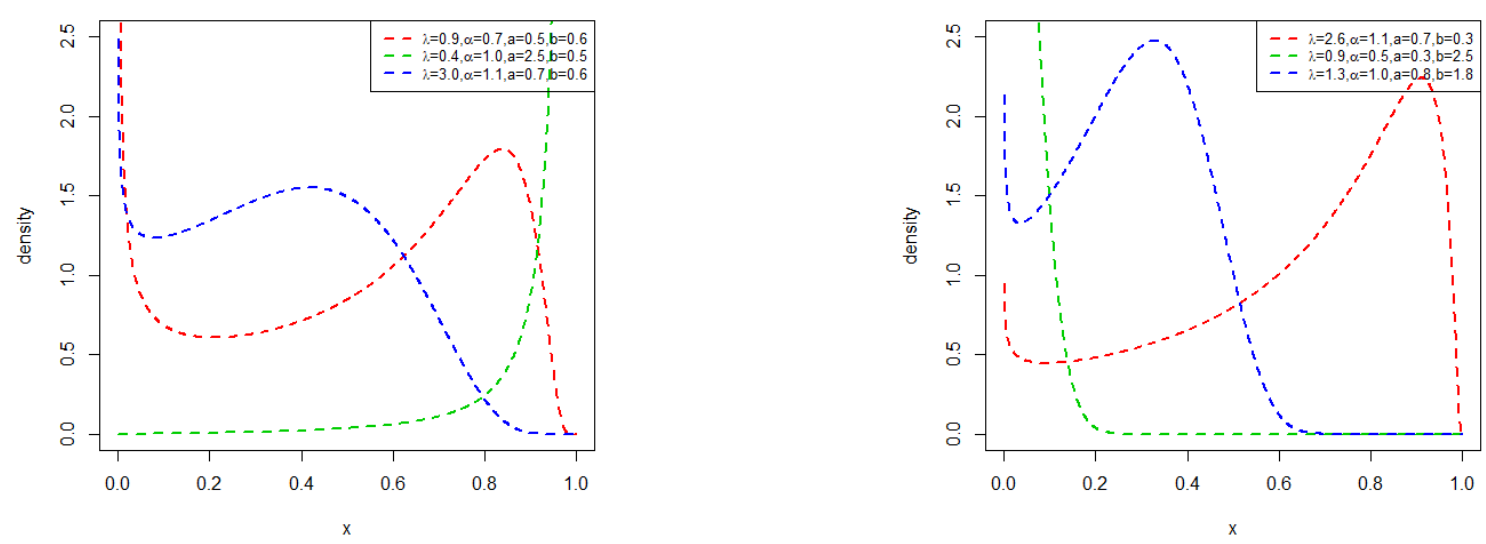

Figure 5: Plots of EHLOL-Kum density function.
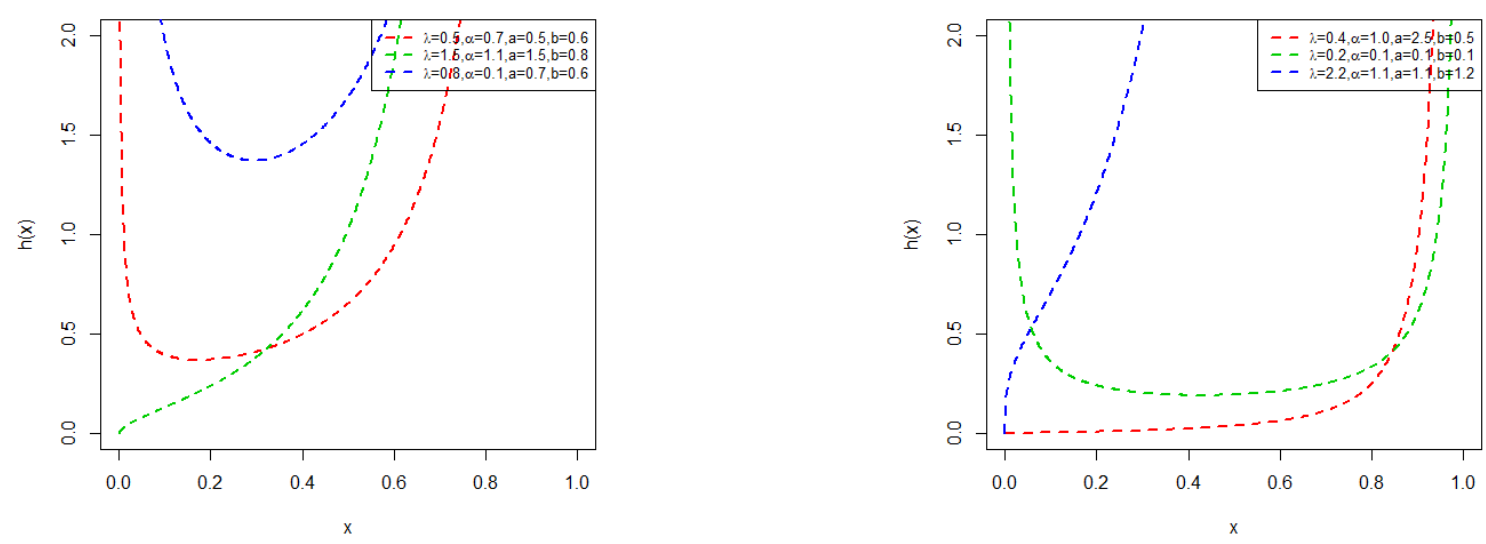

Figure 6: Plots of EHLOL-Kum hazard rate function.

Figure 6 shows the plots of the hrf of the EHLOL-Kum distribution for different parameter values. Graphs of the hrf exhibits increasing and bathtub shapes.

\subsubsection{EHLOL-Fréchet Distribution}

Using the Frétchet distribution with cdf and pdf given by $G(x ; a, b)=\exp \left[-\left(\frac{a}{x}\right)^{b}\right]$ and $g(x ; a, b)=$ 
$b a^{b} x^{-(b+1)} \exp \left[-\left(\frac{a}{x}\right)^{b}\right]$ for $a, b>0$ as a baseline distribution, we get the EHLOL-Fréchet (EHLOL-Fr) distribution with cdf and pdf given by

$$
\mathrm{F}_{\text {EHLOL }-\mathrm{Fr}}(\mathrm{x})=\left\{\frac{1-\frac{\lambda+\left(1-\exp \left[-\left(\frac{\mathrm{a}}{\mathrm{x}}\right)^{\mathrm{b}}\right]\right)}{(1+\lambda)\left(1-\exp \left[-\left(\frac{\mathrm{a}}{\mathrm{x}}\right)^{\mathrm{b}}\right]\right)} \exp \left[\frac{-\lambda \exp \left[-\left(\frac{\mathrm{a}}{\mathrm{x}}\right)^{\mathrm{b}}\right]}{\left(1-\exp \left[-\left(\frac{\mathrm{a}}{x}\right)^{\mathrm{b}}\right]\right)}\right]}{1+\frac{\lambda+\left(1-\exp \left[-\left(\frac{\mathrm{a}}{\mathrm{x}}\right)^{\mathrm{b}}\right]\right)}{(1+\lambda)\left(1-\exp \left[-\left(\frac{\mathrm{a}}{\mathrm{x}}\right)^{\mathrm{b}}\right]\right)} \exp \left[\frac{-\lambda \exp \left[-\left(\frac{\mathrm{a}}{\mathrm{x}}\right)^{\mathrm{b}}\right]}{\left(1-\exp \left[-\left(\frac{\mathrm{a}}{x}\right)^{\mathrm{b}}\right]\right)}\right]}\right\}^{\alpha}
$$

and

$$
\begin{aligned}
f_{\text {EHLOL-Fr }}(x)= & \frac{2 \alpha \lambda^{2} b^{b} x^{-(b+1)} \exp \left[-\left(\frac{a}{x}\right)^{b}\right] \exp \left[\frac{-\lambda \exp \left[-\left(\frac{a}{x}\right)^{b}\right]}{\left(1-\exp \left[-\left(\frac{a}{x}\right)^{b}\right]\right)}\right]}{(1+\lambda)\left(1-\exp \left[-\left(\frac{a}{x}\right)^{b}\right]\right)^{3}} \\
& \times \frac{\left\{1-\frac{\lambda+\left(1-\exp \left[-\left(\frac{a}{x}\right)^{b}\right]\right)}{(1+\lambda)\left(1-\exp \left[-\left(\frac{a}{x}\right)^{b}\right]\right)} \exp \left[\frac{-\lambda \exp \left[-\left(\frac{a}{x}\right)^{b}\right]}{\left(1-\exp \left[-\left(\frac{\mathrm{a}}{x}\right)^{b}\right]\right)}\right]\right\}^{\alpha-1}}{\left\{1+\frac{\lambda+\left(1-\exp \left[-\left(\frac{a}{x}\right)^{b}\right]\right)}{(1+\lambda)\left(1-\exp \left[-\left(\frac{a}{x}\right)^{b}\right]\right)} \exp \left[\frac{-\lambda \exp \left[-\left(\frac{a}{x}\right)^{b}\right]}{\left(1-\exp \left[-\left(\frac{a}{x}\right)^{b}\right]\right)}\right]\right\}^{\alpha+1}} .
\end{aligned}
$$

Figure 7 shows the plots of the EHLOL-Fr distribution for different parameter values. The pdf can take various shapes including reverse J, uni-modal, left skewed and right skewed.
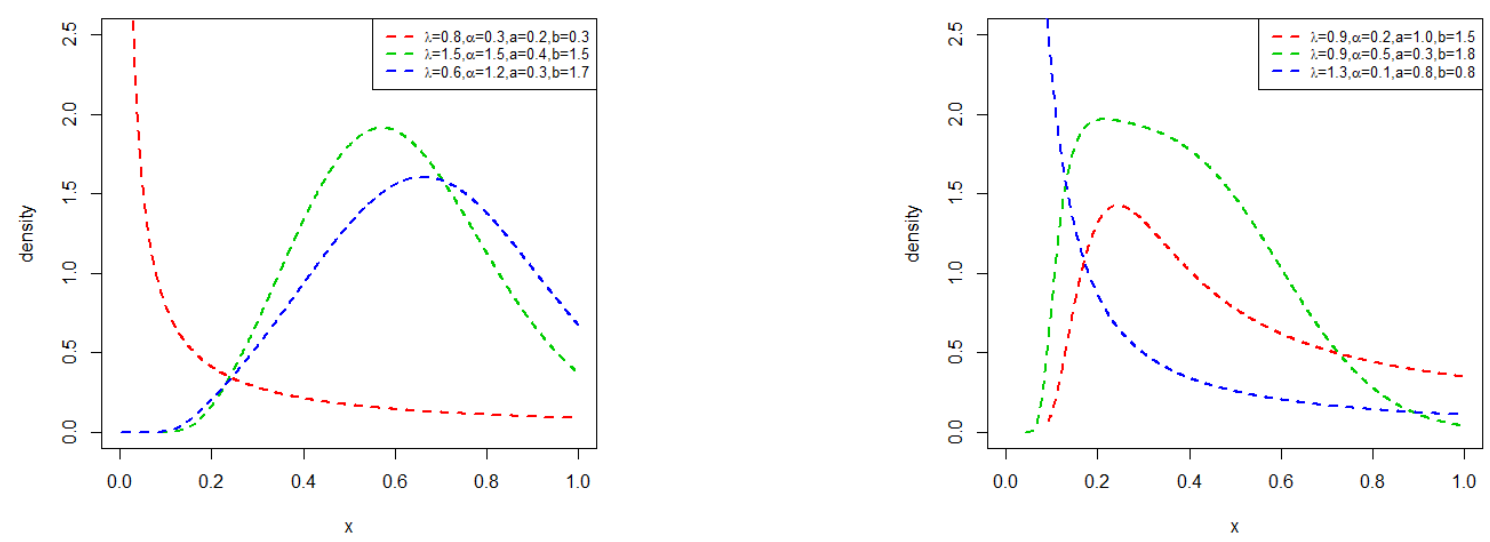

Figure 7: Plots of EHLOL-Fr density function.
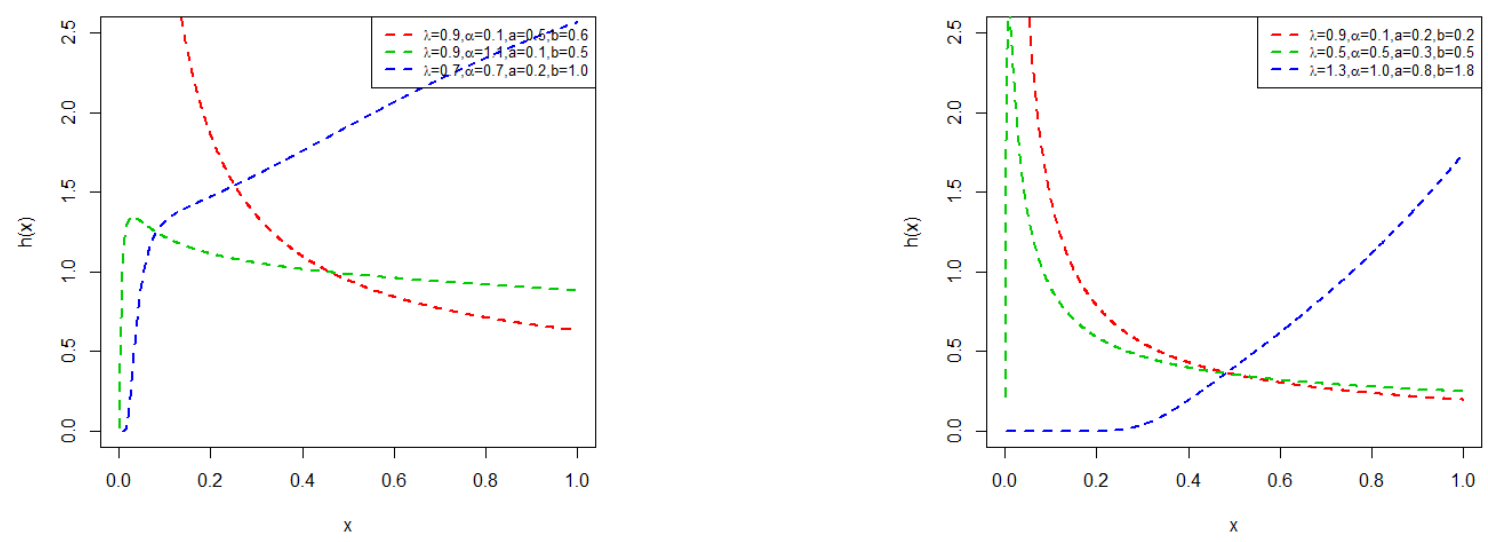

Figure 8: Plots of EHLOL-Fr hazard rate function. 
Figure 8 shows the plots of the hrf of the EHLOL-Fr distribution for different parameter values. Graphs of the hazard rate function exhibits decreasing, increasing and upside-down bathtub shapes.

Table 1 shows some random numbers that are generated from the EHLOL-W distribution for selected parameter values.

Table 1: Table of quantiles for selected parameter values of the EHLOL-W distribution.

\begin{tabular}{cccccc}
\hline \multicolumn{5}{c}{$(\lambda, \alpha, \mathrm{a}, \mathrm{b})$} \\
\hline $\mathrm{u}$ & $(0.8,0.8,0.7,0.5)$ & $(2.0,2.5,0.9,0.5)$ & $(0.5,2.0,1.5,1.5)$ & $(0.9,5.0,1.8,0.5)$ & $(0.8,4.5,1.6,0.4)$ \\
\hline 0.1 & 0.0722 & 0.2117 & 1.7522 & 0.5685 & 0.4744 \\
0.2 & 0.1882 & 0.2761 & 1.9609 & 0.6011 & 0.5060 \\
0.3 & 0.3081 & 0.3250 & 2.0959 & 0.6236 & 0.5276 \\
0.4 & 0.4256 & 0.3679 & 2.2024 & 0.6422 & 0.5455 \\
0.5 & 0.5417 & 0.4090 & 2.2957 & 0.6593 & 0.5619 \\
0.6 & 0.6597 & 0.4508 & 2.3838 & 0.6759 & 0.5779 \\
0.7 & 0.7853 & 0.4962 & 2.4732 & 0.6936 & 0.5948 \\
0.8 & 0.9298 & 0.5504 & 2.5729 & 0.7138 & 0.6142 \\
0.9 & 1.1249 & 0.6272 & 2.7036 & 0.7416 & 0.6408 \\
\hline
\end{tabular}

\section{Moments and incomplete moments}

In this section, moments, moment generating function and incomplete moments are presented.

\subsection{Moments and generating function}

Let $T_{k+l+1}$, denote the Exp-G random variable with power parameter $k+l+1$. Then the $r^{\text {th }}$ raw moment of $X$, say $\mu_{r}^{\prime}$ follows from equation (2.8) as

$$
\mu_{r}^{\prime}=E\left(X^{r}\right)=\sum_{k, l=0}^{\infty} a_{k, l} E\left(T_{k+l+1}^{r}\right),
$$

where $a_{k, l}$ is as given in equation (2.9).

The moment generating function (mgf) $M_{X}(t)=E\left(e^{t X}\right)$ of $X$ can be derived from equation (2.8) and is given by

$$
M_{X}(t)=\sum_{k, l=0}^{\infty} a_{k, l} M_{k+l+1}(t),
$$

where $M_{k+l+1}(t)$ is the mgf of $T_{k+l+1}$. Hence, $M_{X}(t)$ can be determined from the Exp-G generating function.

A table of moments for some selected parameters values of the special case, the EHLOL-Weibull distribution is given in Table 2.

\subsection{Incomplete moments}

Conditional and incomplete moments are useful in the analysis of lifetime data, reliability and in measuring inequality. The $\mathrm{r}^{\text {th }}$ incomplete moment is

$$
\vartheta_{\mathrm{r}}(t)=\sum_{k, l=0}^{\infty} a_{k, l} \int_{-\infty}^{t} x^{r} h_{k+l+1}(x) d x .
$$


Table 2: Table of Moments for the EHLOL-W distribution for selected parameter values.

\begin{tabular}{cccccc}
\hline & $(0.8,0.8,0.7,0.5)$ & $(2.0,2.5,0.9,0.5)$ & $(0.5,2.0,1.5,1.5)$ & $(0.9,5.0,1.8,0.5)$ & $(0.8,4.5,1.6,0.4)$ \\
\hline$E(X)$ & 0.3828 & 0.4155 & 0.0043 & 0.6567 & 0.5592 \\
$E\left(X^{2}\right)$ & 0.2450 & 0.1982 & 0.0036 & 0.4358 & 0.3170 \\
$E\left(X^{3}\right)$ & 0.1778 & 0.1047 & 0.0031 & 0.2922 & 0.1820 \\
$E\left(X^{4}\right)$ & 0.1384 & 0.0599 & 0.0027 & 0.1978 & 0.1058 \\
$E\left(X^{5}\right)$ & 0.1128 & 0.0367 & 0.0024 & 0.1351 & 0.0622 \\
$E\left(X^{6}\right)$ & 0.0948 & 0.0238 & 0.0022 & 0.0931 & 0.0369 \\
$S D$ & 0.0817 & 0.0162 & 0.0020 & 0.0647 & 0.0221 \\
$C V$ & 0.0716 & 0.0115 & 0.0018 & 0.0453 & 0.0134 \\
$C S$ & 0.0636 & 0.0084 & 0.0017 & 0.0319 & 0.0081 \\
$C K$ & 0.0573 & 0.0064 & 0.0016 & 0.0227 & 0.0051 \\
\hline
\end{tabular}

Note that $\vartheta_{1}(t)$ can be applied to construct Bonferroni and Lorenz curves. These curves are of great importance in insurance, demography, reliability, medicine and economics.

A general equation for $\vartheta_{1}(t)$ can be obtained from equation (3.1) as

$$
\vartheta_{1}(t)=\sum_{k, l=0}^{\infty} a_{k, l} P_{k+l+1}(t),
$$

where $P_{k+l+1}(t)=\int_{-\infty}^{t} x h_{k+l+1}(x) d x$ is the first incomplete moment of the Exp-G distribution.

\section{Order statistics, probability weighted moments and entropy}

Order statistics and entropy play important roles in probability and statistics, particularly in reliability, lifetime data analysis and information theory. In this section, we present the distribution of the $i^{\text {th }}$ order statistics and Rényi entropy for the EHLOL-G family of distributions.

\subsection{Order statistics}

Let $X_{1}, X_{2}, \ldots, X_{n}$ be independent and identically distributed EHLOL-G random variables. Then, the pdf of the $i^{\text {th }}$ order statistics $\left(X_{i: n}\right)$ can be written as

$$
f_{i: n}(x)=\frac{f(x)}{B(i, n-i+1)} \sum_{j=0}^{n-i}(-1)^{j}\left(\begin{array}{c}
n-i \\
j
\end{array}\right) F(x)^{j+i-1},
$$

where $B(.,$.$) is the beta function.$

Using equation (2.1) and equation (2.2), we have

$$
\begin{aligned}
f(x) F(x)^{j+i-1}= & \frac{2 \alpha \lambda^{2} g(x ; \underline{\zeta})}{(1+\lambda) \bar{G}^{3}(x ; \underline{\zeta})} \exp \left[\frac{-\lambda G(x ; \underline{\zeta})}{\bar{G}(x ; \underline{\zeta})}\right]\left\{1-\frac{\lambda+\bar{G}(x ; \underline{\zeta})}{(1+\lambda) \bar{G}(x ; \underline{\zeta})} \exp \left[\frac{-\lambda G(x ; \underline{\zeta})}{\bar{G}(x ; \underline{\zeta})}\right]\right\}^{\alpha(j+i)-1} \\
& \times\left\{1+\frac{\lambda+\bar{G}(x ; \underline{\zeta})}{(1+\lambda) \bar{G}(x ; \underline{\zeta})} \exp \left[\frac{-\lambda G(x ; \underline{\zeta})}{\bar{G}(x ; \underline{\zeta})}\right]\right\}^{-\alpha(j+i)-1} .
\end{aligned}
$$

After applying the generalized binomial and the exponential series, we obtain

$$
\begin{aligned}
f(x) F(x)^{j+i-1}= & \sum_{w, s, k, l=0}^{\infty} \sum_{q=0}^{w+s} \frac{2 \alpha g(x ; \zeta)(-1)^{s+k+l} \lambda^{k+w+s+2}}{k !(1+\lambda)^{w+s+1}(w+s+1)^{-k}}\left(\begin{array}{c}
w+s \\
q
\end{array}\right) \\
& \times\left(\begin{array}{c}
\alpha(j+i)-1 \\
s
\end{array}\right)\left(\begin{array}{c}
-\alpha(j+i)-1 \\
w
\end{array}\right)\left(\begin{array}{c}
-(k+q+3) \\
l
\end{array}\right) G(x ; \underline{\zeta})^{k+l} .
\end{aligned}
$$


Substituting equation (4.2) in equation (4.1), the pdf of $X_{i: n}$ can be expressed as

$$
f_{i: n}(x)=\sum_{k, l=0}^{\infty} b_{k, l} h_{k+l+1}(x),
$$

where $h_{k+l+1}(x)$ is the Exp-G density with power parameter $(k+l+1)$ and

$$
\begin{aligned}
b_{k, l}= & \sum_{w, s=0}^{\infty} \sum_{j=0}^{n-i} \sum_{q=0}^{w+s} \frac{2 \alpha(-1)^{j+s+k+l} \lambda^{k+w+s+2}(w+s+1)^{k}}{k !(1+\lambda)^{w+s+1} B(i, n-i+1)(k+l+1)}\left(\begin{array}{c}
n-i \\
j
\end{array}\right) \\
& \times\left(\begin{array}{c}
\alpha(j+i)-1 \\
s
\end{array}\right)\left(\begin{array}{c}
-\alpha(j+i)-1 \\
w
\end{array}\right)\left(\begin{array}{c}
w+s \\
q
\end{array}\right)\left(\begin{array}{c}
-(k+q+3) \\
l
\end{array}\right) .
\end{aligned}
$$

Note that, the density function of the EHLOL-G order statistics is a mixture of Exp-G densities. From the last equation, we note that the properties of $X_{i: n}$ follows from those of $T_{k+l+1}$. Thus, the $q^{\text {th }}$ moments of $X_{i: n}$ can be expressed as

$$
E\left(X_{i: n}^{q}\right)=\sum_{k, l=0}^{\infty} b_{k, l} E\left(T_{k+l+1}^{q}\right) .
$$

\subsection{Probability weighted moments}

The probability weighted moment $(\mathrm{PWM})$ is the expectation of a function of a random variable given that the mean of the variable exists. The $(j, i)^{\text {th }}$ PWM of a random variable $X \sim \operatorname{EHLOL}-G(x)$, say $\wp_{j, i}$ is given by

$$
\wp_{j, i}=E\left(X^{j} F(X)^{i}\right)=\int_{-\infty}^{\infty} x^{j} f(x) F(x)^{i} d x
$$

From equation (4.2),

$$
\begin{aligned}
f(x) F(x)^{i}= & \sum_{w, s, k, l=0}^{\infty} \sum_{q=0}^{w+s} \frac{2 \alpha g(x ; \xi)(-1)^{s+k+l} \lambda^{k+w+s+2}}{k !(1+\lambda)^{w+s+1}(w+s+1)^{-k}}\left(\begin{array}{c}
w+s \\
q
\end{array}\right) \\
& \times\left(\begin{array}{c}
\alpha(i+1)-1 \\
s
\end{array}\right)\left(\begin{array}{c}
-\alpha(i+1)-1 \\
w
\end{array}\right)\left(\begin{array}{c}
-(k+q+3) \\
l
\end{array}\right) G(x ; \underline{\zeta})^{k+l},
\end{aligned}
$$

which can be written as

$$
f(x) F(x)^{i}=\sum_{k, l=0}^{\infty} d_{k, l} h_{k+l+1}(x)
$$

where

$$
\begin{aligned}
d_{k, l}= & \sum_{w, s, k, l=0}^{\infty} \sum_{q=0}^{w+s} \frac{2 \alpha(-1)^{s+k+l} \lambda^{k+w+s+2}}{k !(1+\lambda)^{w+s+1}(w+s+1)^{-k}(k+l+1)}\left(\begin{array}{c}
w+s \\
q
\end{array}\right) \\
& \times\left(\begin{array}{c}
\alpha(i+1)-1 \\
s
\end{array}\right)\left(\begin{array}{c}
-\alpha(\mathfrak{i}+1)-1 \\
w
\end{array}\right)\left(\begin{array}{c}
-(k+q+3) \\
l
\end{array}\right) .
\end{aligned}
$$

Consequently the PWM of $X$ is given by

$$
\wp_{j, i}=\sum_{k, l=0}^{\infty} d_{k, l} \int_{-\infty}^{\infty} x^{j} h_{k+l+1}(x) d x=\sum_{k, l=0}^{\infty} d_{k, l} E\left(T_{k+l+1}^{j}\right) .
$$




\subsection{Rényi entropy}

Rényi entropy ([13]) is an extension of Shannon entropy. Rényi entropy is defined to be

$$
I_{R}(v)=\frac{1}{1-v} \log \left(\int_{0}^{\infty}[f(x ; \lambda, \alpha, \underline{\zeta})]^{v} d x\right), v \neq 1, v>0 .
$$

Set $[f(x ; \lambda, \alpha, \underline{\zeta})]^{\nu}=[f(x)]^{\nu}$. Using the pdf (2.2), we can write

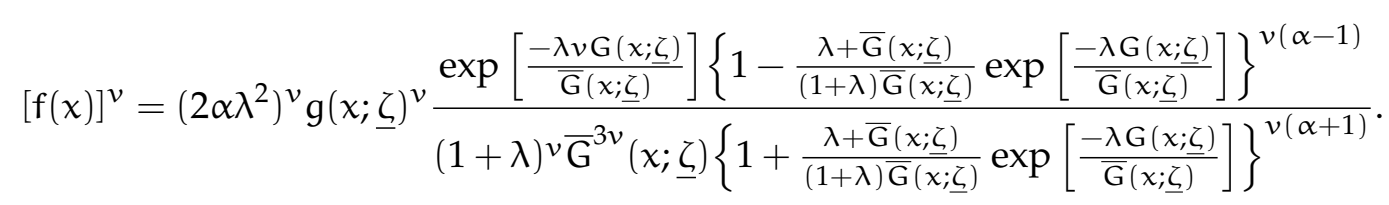

By applying the generalized binomial series expansion, the last equation can be written as:

$$
\begin{aligned}
{[f(x)]^{v}=} & \frac{\left(2 \alpha \lambda^{2}\right)^{v} g(x ; \underline{\zeta})^{v}}{(1+\lambda)^{v} \bar{G}^{3 v}(x ; \underline{\zeta})} \sum_{j, i=0}^{\infty}(-1)^{j}\left(\begin{array}{c}
v(\alpha-1) \\
j
\end{array}\right)\left(\begin{array}{c}
-v(\alpha+1) \\
i
\end{array}\right)\left[\frac{\lambda+\bar{G}(x ; \underline{\zeta})}{(1+\lambda) \bar{G}(x ; \underline{\zeta})}\right]^{j+i} \\
& \times \exp \left[\frac{-\lambda(j+i+v) G(x ; \underline{\zeta})}{\bar{G}(x ; \underline{\zeta})}\right] .
\end{aligned}
$$

From the exponential series, we can write

$$
\begin{aligned}
{[f(x)]^{v}=} & (2 \alpha)^{v} g(x ; \underline{\zeta})^{v} \sum_{j, i, k=0}^{\infty} \sum_{q=0}^{j+i} \frac{(-1)^{j+k} \lambda^{j+i+k+2 v}(j+i+v)^{k}}{k !(1+\lambda)^{j+i+v}}\left(\begin{array}{c}
v(\alpha-1) \\
j
\end{array}\right) \\
& \times\left(\begin{array}{c}
-v(\alpha+1) \\
i
\end{array}\right)\left(\begin{array}{c}
j+i \\
q
\end{array}\right) G(x ; \underline{\zeta})^{k} \bar{G}(x ; \underline{\zeta})^{-(k+q+3 v)} .
\end{aligned}
$$

Using the generalized binomial series expansion in equation (2.7), we have

$$
\begin{aligned}
{[f(x)]^{v}=} & \sum_{j, i, k, l=0}^{\infty} \sum_{q=0}^{j+i} \frac{(2 \alpha)^{v}(-1)^{j+k+1} \lambda^{j+i+k+2 v}(j+i+v)^{k}}{k !(1+\lambda)^{j+i+v}}\left(\begin{array}{c}
v(\alpha-1) \\
j
\end{array}\right) \\
& \times\left(\begin{array}{c}
-v(\alpha+1) \\
i
\end{array}\right)\left(\begin{array}{c}
j+i \\
q
\end{array}\right)\left(\begin{array}{c}
-(k+q+3 v) \\
l
\end{array}\right) g(x ; \zeta)^{v} G(x ; \underline{\zeta})^{k+l}
\end{aligned}
$$

Then, Rényi entropy is given by

$$
\mathrm{I}_{\mathrm{R}}(v)=\frac{1}{1-v} \log \left[\sum_{k, l=0}^{\infty} \mathrm{c}_{k, \mathrm{l}}\left(\int_{0}^{\infty}\left[\mathrm{g}(\mathrm{x} ; \underline{\zeta})^{v} \mathrm{G}(\mathrm{x} ; \underline{\zeta})^{\mathrm{k}+\mathrm{l}}\right] \mathrm{d} x\right)\right],
$$

where

$$
\begin{aligned}
c_{k, l}= & \sum_{j, i=0}^{\infty} \sum_{q=0}^{j+i} \frac{(2 \alpha)^{v}(-1)^{j+k+l} \lambda^{j+i+k+2 v}(j+i+v)^{k}}{k !(1+\lambda)^{j+i+v}}\left(\begin{array}{c}
v(\alpha-1) \\
j
\end{array}\right) \\
& \times\left(\begin{array}{c}
-v(\alpha+1) \\
i
\end{array}\right)\left(\begin{array}{c}
j+i \\
q
\end{array}\right)\left(\begin{array}{c}
-(k+q+3 v) \\
l
\end{array}\right)
\end{aligned}
$$

and $\int_{0}^{\infty} g(x ; \underline{\zeta})^{v} G(x ; \zeta)^{k+l} d x$ can be numerically obtained.

Alternatively, the Rényi entropy of the EHLOL-G family of distributions can be expressed as

$$
\mathrm{I}_{\mathrm{R}}(v)=\frac{1}{1-v} \log \left[\sum_{\mathrm{k}, \mathrm{l}=0}^{\infty} \mathrm{d}_{\mathrm{k}, \mathrm{l}} \mathrm{e}^{(1-v) \mathrm{I}_{\mathrm{REG}}}\right],
$$


where

$$
\begin{aligned}
d_{k, l}= & \sum_{j, i=0}^{\infty} \sum_{q=0}^{j+i} \frac{(2 \alpha)^{v}(-1)^{j+k+l} \lambda^{j+i+k+2 v}(j+i+v)^{k}}{k !(1+\lambda)^{j+i+v}}\left(\begin{array}{c}
v(\alpha-1) \\
j
\end{array}\right) \\
& \times\left(\begin{array}{c}
-v(\alpha+1) \\
i
\end{array}\right)\left(\begin{array}{c}
j+i \\
q
\end{array}\right)\left(\begin{array}{c}
-(k+q+3 v) \\
l
\end{array}\right)\left(\frac{k+l}{v}+1\right)
\end{aligned}
$$

and $I_{R E G}=\int_{0}^{\infty}\left[\left(\frac{k+l}{v}+1\right) g(x ; \underline{\zeta}) G(x ; \underline{\zeta})^{\frac{k+l}{v}}\right]^{v} d x$ is the Rényi entropy of the Exp-G distribution with power parameter $\frac{k+l}{v}$. Thus, the Rényi entropy of the EHLOL-G family of distributions can be obtained directly from that of the Exp-G distribution.

\section{Maximum likelihood estimation}

In this section, we present the method of maximum likelihood for estimating the parameters of the EHLOL-G family of distributions. Let $X \sim \operatorname{EHLOL}-\mathrm{G}(\lambda, \alpha, \underline{\zeta})$ and $\Delta=(\lambda, \alpha, \underline{\zeta})^{\mathrm{T}}$ be the vector of model parameters, then the log-likelihood function $\ell_{n}=\ell_{n}(\Delta)$ based on a random sample of size $n$ from the EHLOL-G family of distributions is given by

$$
\begin{aligned}
\ell_{\mathrm{n}}(\Delta)= & n \log (2 \alpha)+2 \mathrm{n} \log (\lambda)-\mathrm{n} \log (1+\lambda)+\sum_{i=1}^{\mathrm{n}} \log \left(\mathrm{g}\left(\mathrm{x}_{i} ; \underline{\zeta}\right)\right)-3 \sum_{i=1}^{\mathrm{n}} \log \left(\overline{\mathrm{G}}\left(\mathrm{x}_{i} ; \underline{\zeta}\right)\right) \\
& -\lambda \sum_{i=1}^{\mathrm{n}} \frac{\mathrm{G}\left(x_{i} ; \underline{\zeta}\right)}{\overline{\mathrm{G}}\left(x_{i} ; \underline{\zeta}\right)}+(\alpha-1) \sum_{i=1}^{n} \log \left\{1-\frac{\lambda+\overline{\mathrm{G}}\left(x_{i} ; \underline{\zeta}\right)}{(1+\lambda) \overline{\mathrm{G}}\left(x_{i} ; \underline{\zeta}\right)} \exp \left[\frac{-\lambda \mathrm{G}\left(x_{i} ; \underline{\zeta}\right)}{\overline{\mathrm{G}}\left(x_{i} ; \underline{\zeta}\right)}\right]\right\} \\
& -(\alpha+1) \sum_{i=1}^{n} \log \left\{1+\frac{\lambda+\overline{\mathrm{G}}\left(x_{i} ; \underline{\zeta}\right)}{(1+\lambda) \overline{\mathrm{G}}\left(x_{i} ; \underline{\zeta}\right)} \exp \left[\frac{-\lambda \mathrm{G}\left(x_{i} ; \underline{\zeta}\right)}{\overline{\mathrm{G}}\left(x_{i} ; \underline{\zeta}\right)}\right]\right\} .
\end{aligned}
$$

The first derivative of the log-likelihood function with respect to each component of the parameter vector $\Delta=(\lambda, \alpha, \zeta)^{\top}$, that is, elements of the score vector $\mathrm{U}(\Delta)$ are given in the appendix

The maximum likelihood estimates of the parameters, denoted by $\hat{\Delta}$ is obtained by solving the nonlinear equation $\left(\frac{\partial \ell_{n}}{\partial \lambda}, \frac{\partial \ell_{n}}{\partial \alpha}, \frac{\partial \ell_{n}}{\partial \underline{\zeta}_{k}}\right)^{\top}=\mathbf{0}$, using a numerical method such as Newton-Raphson procedure. The multivariate normal distribution $\mathrm{N}_{\mathrm{p}+2}\left(\underline{0}, \mathrm{~J}(\hat{\Delta})^{-1}\right)$, where the mean vector $\underline{\mathbf{0}}=(0,0, \underline{0})^{\top}$ and $\mathrm{J}(\hat{\Delta})^{-1}$ is the observed Fisher information matrix evaluated at $\hat{\Delta}$, can be used to construct confidence intervals and confidence regions for the individual model parameters and for the survival and hazard rate functions.

\section{Simulation study}

We simulate $N=1000$ samples for the true parameters values given in Tables 3 and 4 . The table lists the mean MLEs of the model parameters along with the respective bias and root mean squared errors (RMSEs). The bias and RMSE for the estimated parameter, say, $\hat{\theta}$, say, are given by:

$$
\operatorname{Bias}(\hat{\theta})=\frac{\sum_{i=1}^{N} \hat{\theta}_{i}}{N}-\theta, \quad \text { and } \operatorname{RMSE}(\hat{\theta})=\sqrt{\frac{\sum_{i=1}^{N}\left(\hat{\theta}_{i}-\theta\right)^{2}}{N}},
$$

respectively.

The results in Tables 3 and 4 clearly show that the mean estimates of the parameters tend to be closer to the true parameter values as the sample size $n$ increases, also the bias and the RMEs are decaying towards zero which implies strong consistency of the MLE of the model. 
Table 3: Simulation results.

\begin{tabular}{|c|c|c|c|c|c|c|c|}
\hline & & \multicolumn{3}{|c|}{$(0.5,0.5,0.9,0.8)$} & \multicolumn{3}{|c|}{$(0.5,0.7,0.5,0.8)$} \\
\hline parameter & Sample Size & Mean & RMSE & Bias & Mean & RMSE & Bias \\
\hline \multirow[t]{5}{*}{$\lambda$} & 50 & 1.6430 & 8.2837 & 1.1430 & 5.7617 & 29.4288 & 5.2617 \\
\hline & 100 & 1.3057 & 5.4868 & 0.8057 & 2.4042 & 7.7581 & 1.9042 \\
\hline & 200 & 0.7384 & 0.5207 & 0.2384 & 1.3829 & 3.2974 & 0.8829 \\
\hline & 400 & 0.6821 & 0.3055 & 0.1821 & 1.0200 & 0.6966 & 0.5200 \\
\hline & 800 & 0.6270 & 0.2043 & 0.1270 & 0.8646 & 0.4600 & 0.3646 \\
\hline \multirow[t]{5}{*}{$\alpha$} & 50 & 0.4505 & 0.1984 & -0.0495 & 0.6332 & 0.3021 & -0.0668 \\
\hline & 100 & 0.4564 & 0.1412 & -0.0436 & 0.6437 & 0.2196 & -0.0563 \\
\hline & 200 & 0.4869 & 0.0996 & -0.0131 & 0.6966 & 0.1455 & -0.0034 \\
\hline & 400 & 0.4912 & 0.0687 & -0.0088 & 0.6947 & 0.1018 & -0.0053 \\
\hline & 800 & 0.5042 & 0.0407 & 0.00422 & 0.7040 & 0.0560 & 0.0039 \\
\hline \multirow[t]{5}{*}{ a } & 50 & 1.4348 & 1.1274 & 0.5348 & 0.9539 & 0.9003 & 0.4539 \\
\hline & 100 & 1.3055 & 0.8158 & 0.4055 & 0.8504 & 0.6353 & 0.3504 \\
\hline & 200 & 1.1045 & 0.4432 & 0.2045 & 0.6916 & 0.3575 & 0.1916 \\
\hline & 400 & 1.0506 & 0.2852 & 0.1506 & 0.6740 & 0.2556 & 0.1740 \\
\hline & 800 & 0.9822 & 0.1564 & 0.0822 & 0.6231 & 0.1577 & 0.1231 \\
\hline \multirow[t]{5}{*}{$b$} & 50 & 1.2024 & 0.9284 & 0.4024 & 4.8867 & 18.5952 & 4.0867 \\
\hline & 100 & 1.1668 & 0.7777 & 0.3668 & 3.1189 & 4.5684 & 2.3189 \\
\hline & 200 & 1.0364 & 0.4479 & 0.2364 & 2.1833 & 2.3932 & 1.3833 \\
\hline & 400 & 1.0135 & 0.3510 & 0.2135 & 2.0223 & 1.5740 & 1.2223 \\
\hline & 800 & 0.9466 & 0.2457 & 0.1466 & 1.6828 & 1.1068 & 0.8828 \\
\hline
\end{tabular}

Table 4: Simulation results.

\begin{tabular}{|c|c|c|c|c|c|c|c|}
\hline & & \multicolumn{3}{|c|}{$(0.2,0.5,0.7,0.4)$} & \multicolumn{3}{|c|}{$(0.5,0.7,0.7,0.8)$} \\
\hline Parameter & Sample size & Mean & RMSE & Bias & Mean & RMSE & Bias \\
\hline \multirow[t]{5}{*}{$\lambda$} & 50 & 0.8839 & 2.0789 & 0.6839 & 3.2587 & 12.2905 & 2.7587 \\
\hline & 100 & 0.8111 & 3.2274 & 0.6111 & 2.6873 & 11.4352 & 2.1873 \\
\hline & 200 & 0.4426 & 0.5011 & 0.2426 & 1.0587 & 2.4737 & 0.5587 \\
\hline & 400 & 0.3887 & 0.2455 & 0.1887 & 0.7260 & 0.4543 & 0.2260 \\
\hline & 800 & 0.3409 & 0.1650 & 0.1409 & 0.6433 & 0.2660 & 0.1433 \\
\hline \multirow[t]{5}{*}{$\alpha$} & 50 & 0.5008 & 0.2096 & 0.0008 & 0.5963 & 0.3149 & -0.1036 \\
\hline & 100 & 0.5089 & 0.1366 & 0.0089 & 0.6259 & 0.2114 & -0.0741 \\
\hline & 200 & 0.5339 & 0.1014 & 0.0339 & 0.6757 & 0.1473 & -0.0243 \\
\hline & 400 & 0.5358 & 0.0728 & 0.0358 & 0.6816 & 0.0925 & -0.0184 \\
\hline & 800 & 0.5344 & 0.0503 & 0.0344 & 0.6972 & 0.0582 & -0.0027 \\
\hline \multirow[t]{5}{*}{ a } & 50 & 1.2039 & 1.0579 & 0.5039 & 1.3711 & 1.3420 & 0.6711 \\
\hline & 100 & 1.0639 & 0.7010 & 0.3660 & 1.1018 & 0.8451 & 0.4018 \\
\hline & 200 & 0.8925 & 0.3998 & 0.1925 & 0.8851 & 0.4692 & 0.1851 \\
\hline & 400 & 0.8592 & 0.2303 & 0.1593 & 0.8210 & 0.2540 & 0.1210 \\
\hline & 800 & 0.8209 & 0.1470 & 0.1209 & 0.7711 & 0.1397 & 0.0711 \\
\hline \multirow[t]{5}{*}{$\mathrm{b}$} & 50 & 0.9814 & 0.9640 & 0.5814 & 1.9460 & 2.5301 & 1.1460 \\
\hline & 100 & 0.9043 & 0.8034 & 0.5043 & 1.6719 & 1.8813 & 0.8720 \\
\hline & 200 & 0.7237 & 0.4966 & 0.3237 & 1.2543 & 0.9739 & 0.4543 \\
\hline & 400 & 0.6971 & 0.3766 & 0.2971 & 1.1344 & 0.6190 & 0.3344 \\
\hline & 800 & 0.6318 & 0.2698 & 0.2318 & 1.0264 & 0.4047 & 0.2264 \\
\hline
\end{tabular}

\section{Applications}

In this section, we present examples to illustrate the flexibility and usefulness of the EHLOL-W distribution and its sub-models for data modeling. The EHLOL-W distribution is fitted to two data sets and 
these fits are compared to the fits of the Odd Log-Logistic Exponentiated Weibull (OLLE-W) distribution ([1]), Kumaraswamy Weibull (KumW) dustribution ([8]), Exponentiated Weibull Lindley (EWL) distribution ([5]), Weibull Lomax (WL) distribution ([15]) and the Beta Generalized Lindley (BGL) distribution ([12]). The pdfs of these distributions are

$$
f_{\mathrm{OLLE}}-W(x)=\frac{\theta \beta \gamma \chi^{\beta-1} e^{-\left(\frac{x}{\alpha}\right)^{\beta}}\left[1-e^{-\left(\frac{x}{\alpha}\right)^{\beta}}\right]^{\gamma \theta-1}\left\{1-\left[1-e^{-\left(\frac{x}{\alpha}\right)^{\beta}}\right]^{\alpha}\right\}^{\theta-1}}{\alpha^{\beta}\left\{\left[1-e^{-\left(\frac{x}{\alpha}\right)^{\beta}}\right]^{\gamma \theta}+\left\{1-\left[1-e^{-\left(\frac{x}{\alpha}\right)^{\beta}}\right]^{\gamma}\right\}^{\theta}\right\}^{2}},
$$

for $\alpha, \beta, \theta, \gamma>0$ and $x>0$,

$$
f_{K u m-W}(x)=a b \beta \alpha^{\beta} x^{\beta-1} e^{-(\alpha x)^{\beta}}\left(1-e^{-(\alpha x)^{\beta}}\right)^{a-1}\left\{1-\left[1-e^{-(\alpha x)^{\beta}}\right]^{a}\right\}^{b-1},
$$

for $\alpha, \beta, a, b>0$ and $x>0$,

$$
\begin{aligned}
f_{E W L}(x)= & \frac{a b v(1+\alpha) \alpha^{2}(1+x) \exp (\alpha x)}{(1+\alpha+\alpha x)^{2}} \exp \left\{-a\left[\frac{(1+\alpha) \exp (\alpha x)}{1+\alpha+\alpha x}-1\right]^{b}\right\} \\
& \times\left(1-\exp \left\{-a\left[\frac{(1+\alpha) \exp (\alpha x)}{1+\alpha+\alpha x}-1\right]\right\}\right)^{v-1}\left\{\frac{(1+\alpha) \exp (\alpha x)}{1+\alpha+\alpha x}-1\right\}^{b-1},
\end{aligned}
$$

for $\alpha, v, a, b>0$ and $x>0$,

$$
f_{W L}(x)=\frac{a b \alpha}{\beta}\left[1+\left(\frac{x}{\beta}\right)\right]^{b \alpha-1}\left\{1-\left[1+\left(\frac{x}{\beta}\right)\right]^{-\alpha}\right\}^{b-1} \exp \left\{-a\left\{\left[1+\left(\frac{x}{\beta}\right)\right]^{\alpha}-1\right\}\right\},
$$

for $\alpha, \beta, a, b>0$ and $x>0$,

$$
f_{B G L}(x)=\frac{\alpha \theta^{2}(1+x) e^{-\theta x}}{B(a, b)(1+\theta)}\left[1-\frac{1+\theta+\theta x}{1+\theta} e^{-\theta x}\right]^{a \alpha-1}\left[1-\left(1-\frac{1+\theta+\theta x}{1+\theta} e^{-\theta x}\right)^{a}\right]^{b-1},
$$

for $\alpha, \theta, a, b>0$ and $x>0$.

Plots of the fitted densities, the histogram of the data and probability plots ([3]) are given in Figures 9 and 10. For the probability plot, we plotted $F\left(x_{(j)} ; \hat{\lambda}, \hat{\alpha}, \underline{\hat{\zeta}}\right)$ against $\frac{j-0.375}{n+0.25}, j=1,2, \ldots, n$, where $x_{(j)}$ are the ordered values of the observed data. The measure of closeness was obtained and is given by the sum of squares

$$
S S=\sum_{j=1}^{n}\left[F\left(x_{(j)} ; \hat{\lambda}, \hat{\alpha}, \underline{\hat{\zeta}}\right)-\left(\frac{j-0.375}{n+0.25}\right)\right]^{2} .
$$

The goodness-of-fit statistics $W^{*}$ and $A^{*}$, see ([4]) are also presented in the tables. These statistics can be used to verify which distribution fits better to the data. In general, the smaller the values of $W^{*}$ and $A^{*}$, the better the fit.

The MLEs of the parameters with standard errors in parenthesis and the values of the statistics ($2 \log (\mathrm{L}), \mathrm{AIC}, \mathrm{AICC}, \mathrm{BIC}, \mathrm{W}^{*}, \mathrm{~A}^{*}, \mathrm{KS}$ and its P-Value) are given in Tables 5 and 6.

\subsection{Relief times of 20 patients}

This data set taken from [11] gives the relief times of 20 patients receiving an analgesic. The estimated parameters, standard error (in parenthesis) of estimates and the goodness-of-fit statistics are presented in Table 5. 
Table 5: Estimation of the EHLOL-W model for relief times of 20 patients data set.

\begin{tabular}{|c|c|c|c|c|c|c|c|c|c|c|c|c|c|}
\hline & & & Estimates & & & & & & & & & & \\
\hline Distribution & $\lambda$ & $\alpha$ & $\mathrm{a}$ & $\mathrm{b}$ & $-2 \log \mathrm{L}$ & AIC & CAIC & BIC & $W^{*}$ & $A^{*}$ & $\mathrm{~K}-\mathrm{S}$ & $\mathrm{P}$-value & SS \\
\hline EHLOL-W & $\begin{array}{l}24.355 \\
(5.714)\end{array}$ & $\begin{array}{c}8265.200 \\
(0.001)\end{array}$ & $\begin{array}{c}0.335 \\
(0.069) \\
\end{array}$ & $\begin{array}{l}37.842 \\
(2.028)\end{array}$ & 31.065 & 39.065 & 41.731 & 43.048 & 0.033 & 0.188 & 0.103 & 0.984 & 0.023 \\
\hline BGL & $\begin{array}{c}\alpha \\
0.120 \\
(0.013)\end{array}$ & $\begin{array}{c}\theta \\
\\
1.520 \times 10^{-07} \\
\left(4.342 \times 10^{-06}\right)\end{array}$ & $\begin{array}{c}\mathrm{a} \\
0.302 \\
(0.001)\end{array}$ & $\begin{array}{c}\mathrm{b} \\
10.100 \\
\left(3.494 \times 10^{-05}\right)\end{array}$ & 162.523 & 170.538 & 173.205 & 174.521 & 0.089 & 0.523 & 0.512 & $5.526 \times 10^{-05}$ & 1.571 \\
\hline OLLE-W & $\begin{array}{c}\alpha \\
0.027 \\
(0.021)\end{array}$ & $\begin{array}{c}\beta \\
0.034 \\
(0.007)\end{array}$ & $\begin{array}{c}\theta \\
90.217 \\
\left(5.119 \times 10^{-05}\right)\end{array}$ & $\begin{array}{c}\gamma \\
1.821 \\
(0.037)\end{array}$ & 32.987 & 40.987 & 43.654 & 44.970 & 0.052 & 0.313 & 0.120 & 0.969 & 0.029 \\
\hline Kum-W & $\begin{array}{c}\mathrm{a} \\
23108.000 \\
\left(9.797 \times 10^{-06}\right) \\
\end{array}$ & $\begin{array}{c}\mathrm{b} \\
1.465 \\
(0.536) \\
\end{array}$ & $\begin{array}{c}c \\
0.309 \\
(0.004) \\
\end{array}$ & $\begin{array}{c}\lambda \\
989.690 \\
\left(2.177 \times 10^{-04}\right) \\
\end{array}$ & 31.319 & 39.319 & 41.986 & 43.302 & 0.036 & 0.207 & 0.121 & 0.931 & 0.032 \\
\hline WL & $\begin{array}{c}\mathrm{a} \\
1753.0 \\
(0.018)\end{array}$ & $\begin{array}{c}\mathrm{b} \\
5.843 \\
(12.087)\end{array}$ & $\begin{array}{c}\alpha \\
0.124 \\
(0.129)\end{array}$ & $\begin{array}{c}\beta \\
0.334 \\
(2.583)\end{array}$ & 38.901 & 46.901 & 49.568 & 50.884 & 0.151 & 0.892 & 0.178 & 0.554 & 0.138 \\
\hline EWL & $\begin{array}{c}\alpha \\
0.003 \\
(0.003)\end{array}$ & $\begin{array}{c}\nu \\
982.470 \\
\left(2.881 \times 10^{-06}\right)\end{array}$ & $\begin{array}{c}\mathrm{a} \\
348.730 \\
\left(1.466 \times 10^{-06}\right)\end{array}$ & $\begin{array}{c}\mathrm{b} \\
0.374 \\
(0.060)\end{array}$ & 31.657 & 39.657 & 42.323 & 43.640 & 0.042 & 0.244 & 0.123 & 0.924 & 0.034 \\
\hline W & & & $\begin{array}{c}\mathrm{a} \\
0.122 \\
(0.056) \\
\end{array}$ & $\begin{array}{c}\mathrm{b} \\
2.787 \\
(0.427) \\
\end{array}$ & 41.173 & 45.173 & 45.879 & 47.164 & 0.186 & 1.093 & 0.185 & 0.501 & 0.171 \\
\hline
\end{tabular}

The estimated variance-covariance matrix for the relief times of 20 patients data is given by,

$$
\left[\begin{array}{cccc}
32.6503 & 0.0046 & 0.3914 & -11.5889 \\
0.0046 & 6.4167 \times 10^{-07} & 5.4864 \times 10^{-05} & -0.0016 \\
0.3914 & 5.4867 \times 10^{-05} & 0.0047 & -0.1389 \\
-11.5889 & -0.0016 & -0.1389 & 4.1133
\end{array}\right]
$$

and the $95 \%$ confidence intervals for the parameters $\lambda, \alpha, a$ and $b$ are: $24.355 \pm 11.199,8265.200 \pm 0.002$, $0.335 \pm 0.135$, and $37.842 \pm 3.975$, respectively.

From the results presented in Table 5, it can be seen that the EHLOL-W distribution has smallest values of the goodness-of-fit statistics (AIC, AICC, BIC, $W^{*}, A^{*}$ ), also the biggest $p$-value and the smallest Sum of Squares value (SS) indicating that the EHLOL-W distribution provides a better fit compared to the other models. The plots of histogram, fitted densities and probability plots are given in Figure 9.
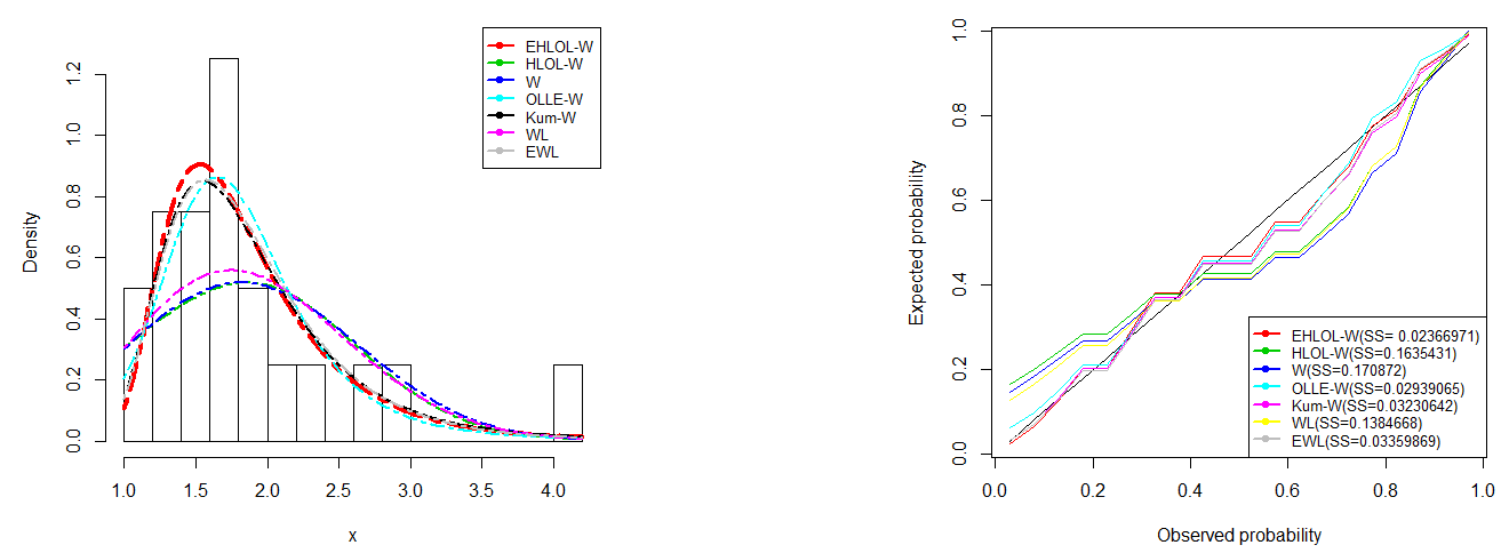

Figure 9: Fitted densities and probability plots for relief times of 20 patients data set.

\subsection{Strengths of glass fibres data set}

The data consists of 63 observations of the strengths of $1.5 \mathrm{~cm}$ glass fibres, originally obtained by workers at the UK National Physical Laboratory. The units of measurement are not given in the paper. [14] also analysed this data set. The estimated parameters, standard error (in parenthesis) of estimates and the goodness-of-fit statistics for this data set are presented in Table 6. 
Table 6: Estimation of the EHLOL-W model for strengths of glass fibres data set.

\begin{tabular}{|c|c|c|c|c|c|c|c|c|c|c|c|c|c|}
\hline & & & Estimates & & & & & & & & & & \\
\hline Distribution & $\lambda$ & $\alpha$ & $a$ & $\mathrm{~b}$ & $-2 \log \mathrm{L}$ & AIC & CAIC & BIC & $W^{*}$ & $A^{*}$ & $\mathrm{~K}-\mathrm{S}$ & $\mathrm{P}$-value & SS \\
\hline EHLOL-W & $\begin{array}{c}0.331 \\
(1.007)\end{array}$ & $\begin{array}{c}1.436 \\
(0.770)\end{array}$ & $\begin{array}{c}1.327 \\
(1.176)\end{array}$ & $\begin{array}{c}0.872 \\
(1.272)\end{array}$ & 26.763 & 34.763 & 35.453 & 43.336 & 0.134 & 0.762 & 0.123 & 0.301 & 0.124 \\
\hline BGL & $\begin{array}{c}\alpha \\
0.121 \\
(0.007)\end{array}$ & $\begin{array}{c}\theta \\
2.290 \times 10^{-06} \\
\left(2.983 \times 10^{-06}\right)\end{array}$ & $\begin{array}{c}\mathrm{a} \\
0.302 \\
\left(4.513 \times 10^{-04}\right)\end{array}$ & $\begin{array}{c}\mathrm{b} \\
0.101 \\
\left(2.218 \times 10^{-05}\right)\end{array}$ & 492.265 & 500.270 & 500.960 & 508.843 & 0.638 & 3.492 & 0.521 & $2.776 \times 10^{-15}$ & 5.151 \\
\hline OLLE-W & $\begin{array}{c}\alpha \\
1.992 \\
(0.297)\end{array}$ & $\begin{array}{c}\beta \\
8.749 \\
(3.940)\end{array}$ & $\begin{array}{c}\theta \\
1.687 \\
(0.744)\end{array}$ & $\begin{array}{c}\gamma \\
0.302 \\
(0.267)\end{array}$ & 28.050 & 36.050 & 36.739 & 44.622 & 0.186 & 1.031 & 0.132 & 0.222 & 0.153 \\
\hline WL & $\begin{array}{c}\mathrm{a} \\
0.013 \\
(0.006) \\
\end{array}$ & $\begin{array}{c}\mathrm{b} \\
2.840 \\
(0.270) \\
\end{array}$ & $\begin{array}{c}\alpha \\
0.010 \\
(0.005) \\
\end{array}$ & $\begin{array}{c}\beta \\
95.349 \\
(0.005) \\
\end{array}$ & 28.792 & 36.792 & 37.481 & 45.365 & 0.173 & 0.974 & 0.136 & 0.193 & 0.167 \\
\hline EWL & $\begin{array}{c}\alpha \\
4.195 \\
(5.940)\end{array}$ & $\begin{array}{c}v \\
1.808 \\
(0.981)\end{array}$ & $\begin{array}{c}a \\
0.020 \\
(0.028)\end{array}$ & $\begin{array}{c}\mathrm{b} \\
0.717 \\
(1.146)\end{array}$ & 28.219 & 36.219 & 36.909 & 44.792 & 0.160 & 0.899 & 0.131 & 0.230 & 0.150 \\
\hline W & & & $\begin{array}{c}a \\
0.060 \\
(0.021)\end{array}$ & $\begin{array}{c}\mathrm{b} \\
5.781 \\
(0.576)\end{array}$ & 30.414 & 34.414 & 34.614 & 38.700 & 0.237 & 1.304 & 0.152 & 0.108 & 0.211 \\
\hline
\end{tabular}

The estimated variance-covariance matrix for the strengths of glass fibers data set is given by,

$$
\left[\begin{array}{llll}
1.0132 & 0.6607 & 1.1548 & 1.2726 \\
0.6607 & 0.5925 & 0.6657 & 0.7803 \\
1.1548 & 0.6658 & 1.3835 & 1.4868 \\
1.2726 & 0.7803 & 1.4868 & 1.6184
\end{array}\right]
$$

and the $95 \%$ confidence intervals for the parameters $\lambda, \alpha, a$ and $b$ are: $0.331 \pm 1.974,1.436 \pm 1.509,1.327$ \pm 2.305 , and $0.872 \pm 2.489$, respectively.
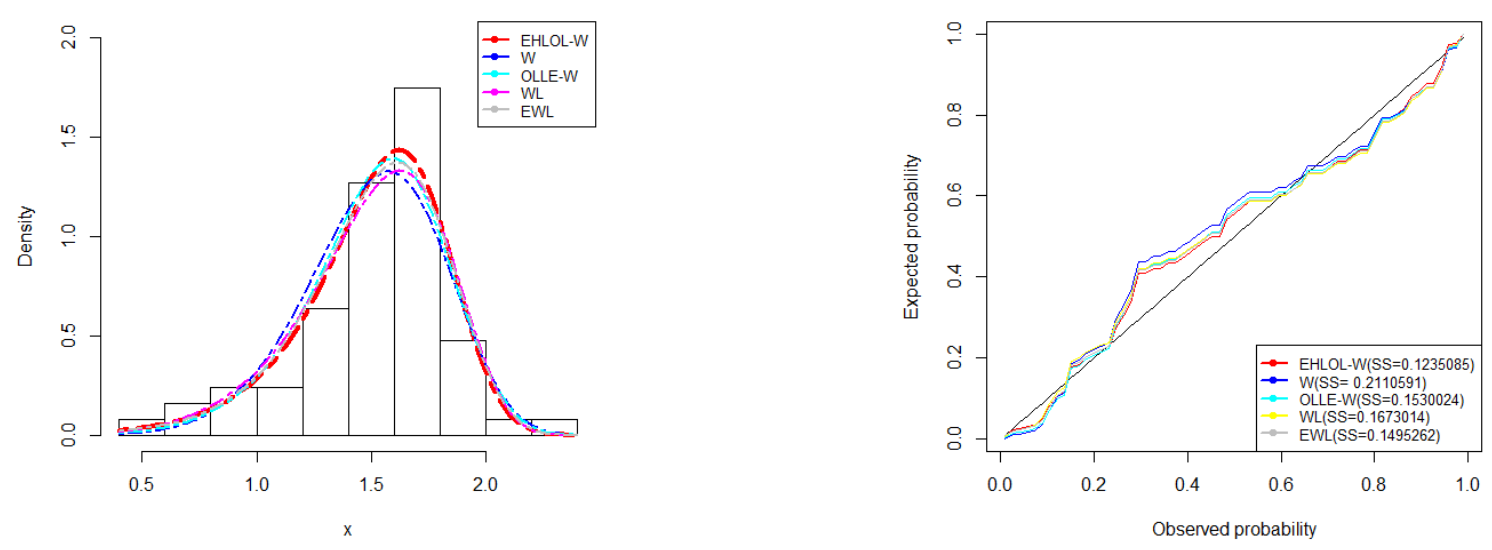

Figure 10: Fitted densities and probability plots for strengths of glass fibers data set.

Figure 10 shows the histogram, fitted densities as well as the probability plots for the strength of glass fibre data set. Clearly, the EHLOL-W distribution has the smallest values of the goodness-of-fit statistics, the largest p-value and the smallest SS value, hence we can conclude that the EHLOL-W distribution provides a better fit compared to the other models presented in Table 6.

\section{Concluding remarks}

A new generalized distribution called the exponentiated Half-Logistic Odd Lindley-G (EHLOL-G) family of distributions is developed and presented. The EHLOL-G family of distributions possesses hazard rate function with flexible behavior. We obtain closed form expressions for the moments, incomplete 
moments, distribution of order statistics and Rényi entropy. Maximum likelihood estimation (MLE) was used to estimate the model parameters. The performance of the special case of the EHLOL-W distribution was examined by conducting various simulations for different sizes. Finally, the special case of the EHLOL-W distribution was fitted to real data sets to illustrate the applicability and usefulness of the proposed family of distributions.

\section{References}

[1] A. Z. Afify, M. Alizadeh, M. Zayed, T. G. Ramires, F. Louzada, The Odd Log-Logistic Exponentiated Weibull Distribution: Regression Modelling, Properties and Applications, Iran. J. Sci. Tech., 42 (2018), 2273-2288. 7

[2] A. Z. Afify, E. Altun, M. Alizadeh, G. Ozel, G. G. Hamedani, The odd exponentiated half-logistic-G family: properties, characterizations and applications, Chil. J. Stat., 8 (2017), 65-91. 1

[3] J. Chambers, W. Cleveland, B. Kleiner, J. Tukey, Graphical Methods for Data Analysis, Chapman and Hall, London, (1983). 7

[4] G. Chen, N. Balakrishnan, A General Purpose Approximate Goodness-of-fit Test, J. Qual. Tech., 27 (1995), 154-161. 7

[5] G. M. Corderio, A. Z. Afify, H. M. Yousof, R. R. Pescim, G. R. Aryal, The Exponentiated Weibull-H Family of Distributions: Theory and Applications, Mediterr. J. Math., 14 (2017), 22 pages. 7

[6] G. M. Cordeiro, M. Alizadeh, P. R. D. Marinho, The Type I Half-Logistic Family of Distributions, J. Stat. Comput. Simul., 86 (2016), 707-728. 1, 2.1

[7] G. M. Cordeiro, M. Alizadeh, E. M. M. Ortega, The Exponentiated Half-Logistic Family of Distributions: Properties and Applications, J. Probab. Stat., 2014 (2014), 21 pages. 1

[8] G. M. Cordeiro, E. M. M. Ortega, S. Nadarajaah, The Kumaraswamy Weibull Distribution with Application to Failure Data, J. Franklin Inst., 347 (2010), 1399-1429. 7

[9] E. El-sayed, E. Mahmoud, Kumaraswamy Type 1 Half Logistic Family of Distributions with Applications, Gazi Unive. J. Sci., 32 (2019), 333-349. 1

[10] F. Gomes-Silva, A. Percontini, E. de Brito, M. W. Ramos, R Venáncio, G. M. Cordeiro, The odd Lindley-G family of distributions, Aust. J. Stat., 46 (2017), 65-87. 2.1

[11] A. J. Gross, V. A. Clark, Survival Distributions: Reliability Applications in the Biomedical Sciences, Wiley, New York, (1975). 7.1

[12] B. O. Oluyede, T. Yang, A New Class of Generalized Lindley Distributions with applcations, J. Stat. Comput. Simul., 85 (2015), 2072-2100. 7

[13] A. Rényi, On Measures of Entropy and Information, Proc. 4th Berkeley Sympos. Math. Statist. and Prob., Univ. California Press, Berkeley, 1961 (1961), 547-561. 4.3

[14] R. L. Smith, J. C. Naylor, A comparison of maximum likelihood and Bayesian estimators for the three-parameter Weibull distribution, J. Roy. Statist. Soc. Ser. C, 36 (1987), 358-369. 7.2

[15] M. H. Tahir, G. M. Cordeiro, M. Mansoor, M. Zubair, The Weibull-Lomax Distribution: Properties and Applications, Hacet. J. Math. Stat., 44 (2015), 461-480. 7

\section{Appendix A. Elements of the score vector}

The elements of the score vector are given by:

$$
\begin{aligned}
& \mathrm{u}_{\alpha}(\Delta)=\frac{\mathrm{n}}{\alpha}+\sum_{i=1}^{\mathrm{n}} \log \left\{1-\frac{\lambda+\overline{\mathrm{G}}\left(\mathrm{x}_{i} ; \underline{\zeta}\right)}{(1+\lambda) \overline{\mathrm{G}}\left(x_{i} ; \underline{\zeta}\right)} \exp \left[\frac{-\lambda \mathrm{G}\left(x_{i} ; \underline{\zeta}\right)}{\overline{\mathrm{G}}\left(x_{i} ; \underline{\zeta}\right)}\right]\right\} \\
& -\sum_{i=1}^{n} \log \left\{1+\frac{\lambda+\bar{G}\left(x_{i} ; \underline{\zeta}\right)}{(1+\lambda) \bar{G}\left(x_{i} ; \underline{\zeta}\right)} \exp \left[\frac{-\lambda G\left(x_{i} ; \underline{\zeta}\right)}{\bar{G}\left(x_{i} ; \underline{\zeta}\right)}\right]\right\} \text {, } \\
& \mathrm{u}_{\lambda}(\Delta)=\frac{2 \mathrm{n}}{\lambda}-\frac{\mathrm{n}}{(1+\lambda)}-\sum_{i=1}^{\mathrm{n}} \frac{\mathrm{G}\left(x_{i} ; \xi\right)}{\overline{\mathrm{G}}\left(x_{i} ; \underline{\zeta}\right)}+(\alpha-1) \\
& \times \sum_{i=1}^{n} \frac{\exp \left[\frac{-\lambda \mathrm{G}\left(x_{i} ; \underline{\zeta}\right)}{\overline{\mathrm{G}}\left(x_{i} ; \underline{\zeta}\right)}\right]\left\{\left(\lambda+\overline{\mathrm{G}} x_{i} ; \underline{\zeta}\right)-(1+\lambda)\left[1-\frac{\mathrm{G}\left(x_{i} ; \underline{\zeta}\right)\left(\lambda+\overline{\mathrm{G}}\left(x_{i} ; \underline{\zeta}\right)\right)}{\overline{\mathrm{G}}\left(x_{i} ; \underline{\zeta}\right)}\right]\right\}}{(1+\lambda)^{2} \overline{\mathrm{G}}\left(x_{i} ; \underline{\zeta}\right)\left\{1-\frac{\lambda+\overline{\mathrm{G}}\left(x_{i} ; \underline{\zeta}\right)}{(1+\lambda) \bar{G}\left(x_{i} ; \underline{\zeta}\right)}\right\}} \\
& +(\alpha+1) \sum_{i=1}^{n} \frac{\exp \left[\frac{-\lambda \mathrm{G}\left(x_{i} ; \underline{\zeta}\right)}{\bar{G}\left(x_{i} ; \underline{\zeta}\right)}\right]\left\{(1+\lambda)\left[1-\frac{\mathrm{G}\left(x_{i} ; \underline{\zeta}\right)\left(\lambda+\overline{\mathrm{G}}\left(x_{i} ; \underline{\zeta}\right)\right)}{\bar{G}\left(x_{i} ; \underline{\zeta}\right)}\right]-\left(\lambda+\overline{\mathrm{G}} x_{i} ; \underline{\zeta}\right)\right\}}{(1+\lambda)^{2} \overline{\mathrm{G}}\left(x_{i} ; \underline{\zeta}\right)\left\{1+\frac{\lambda+\overline{\mathrm{G}}\left(x_{i} ; \underline{\zeta}\right)}{(1+\lambda) \overline{\mathrm{G}}\left(x_{i} ; \underline{\zeta}\right)} \exp \left[\frac{-\lambda \mathrm{G}\left(x_{i} ; \underline{\zeta}\right)}{\overline{\mathrm{G}}\left(x_{i} ; \underline{\zeta}\right)}\right]\right\}}
\end{aligned}
$$


and

$$
\begin{aligned}
& \mathrm{u}_{\underline{\zeta}_{k}}(\Delta)=\sum_{i=1}^{n} \frac{\mathrm{g}^{\prime}\left(x_{i} ; \underline{\zeta}\right)}{\mathrm{g}\left(x_{i} ; \underline{\zeta}\right)}-3 \sum_{i=1}^{\mathrm{n}} \frac{\overline{\mathrm{G}}^{\prime}\left(x_{i} ; \underline{\zeta}\right)}{\overline{\mathrm{G}}\left(x_{i} ; \underline{\zeta}\right)}-\lambda \sum_{i=1}^{\mathrm{n}} \frac{\Psi}{\overline{\mathrm{G}}^{2}\left(x_{i} ; \underline{\zeta}\right)} \\
& +\lambda(\alpha-1) \sum_{i=1}^{n} \frac{\left[\left(\lambda+\overline{\mathrm{G}}\left(x_{i} ; \underline{\zeta}\right)\right) \Psi+\overline{\mathrm{G}}^{\prime}\left(x_{i} ; \underline{\zeta}\right) \overline{\mathrm{G}}\left(x_{i} ; \underline{\zeta}\right)\right] \exp \left[\frac{-\lambda \mathrm{G}\left(x_{i} ; \underline{\zeta}\right)}{\overline{\mathrm{G}}\left(x_{i} ; \underline{\zeta}\right)}\right]}{(1+\lambda) \overline{\mathrm{G}}^{3}\left(x_{i} ; \underline{\zeta}\right)\left\{1-\frac{\lambda+\overline{\mathrm{G}}\left(x_{i} ; \zeta\right)}{(1+\lambda) \overline{\mathrm{G}}\left(x_{i} ; \underline{\zeta}\right)} \exp \left[\frac{-\lambda \mathrm{G}\left(x_{i} ; \underline{\zeta}\right)}{\overline{\mathrm{G}}\left(x_{i} ; \underline{\zeta}\right)}\right]\right\}} \\
& +\lambda(\alpha+1) \sum_{i=1}^{n} \frac{\left[\left(\lambda+\overline{\mathrm{G}}\left(x_{i} ; \underline{\zeta}\right)\right) \Psi+\overline{\mathrm{G}}^{\prime}\left(x_{i} ; \underline{\zeta}\right) \overline{\mathrm{G}}\left(x_{i} ; \underline{\zeta}\right)\right] \exp \left[\frac{-\lambda \mathrm{G}\left(x_{i} ; \underline{\zeta}\right)}{\overline{\mathrm{G}}\left(x_{i} ; \underline{\zeta}\right)}\right]}{(1+\lambda) \overline{\mathrm{G}}^{3}\left(x_{i} ; \underline{\zeta}\right)\left\{1+\frac{\lambda+\overline{\mathrm{G}}\left(x_{i} ; \zeta\right)}{(1+\lambda) \overline{\mathrm{G}}\left(x_{i} ; \underline{\zeta}\right)} \exp \left[\frac{-\lambda \mathrm{G}\left(x_{i} ; \zeta\right)}{\overline{\mathrm{G}}\left(x_{i} ; \underline{\zeta}\right)}\right]\right\}},
\end{aligned}
$$

where $\Psi=\left[\overline{\mathrm{G}}\left(x_{i} ; \underline{\zeta}\right) \mathrm{G}^{\prime}\left(x_{i} ; \underline{\zeta}\right)-\mathrm{G}\left(x_{i} ; \underline{\zeta}\right) \overline{\mathrm{G}}^{\prime}\left(x_{i} ; \underline{\zeta}\right)\right], g^{\prime}\left(x_{i} ; \underline{\zeta}\right)=\frac{\partial g\left(x_{i} ; \underline{\zeta}\right)}{\partial \underline{\zeta}_{k}}, G^{\prime}\left(x_{i} ; \underline{\zeta}\right)=\frac{\partial \mathrm{G}\left(x_{i} ; \underline{\zeta}\right)}{\partial \underline{\zeta}_{k}}$, and $\overline{\mathrm{G}}^{\prime}\left(x_{i} ; \underline{\zeta}\right)=$ $\frac{\partial \overline{\mathrm{G}}\left(\mathrm{x}_{i} ; \underline{\zeta}\right)}{\partial \underline{\zeta}_{k}}$.

\section{Appendix B. R define codes}

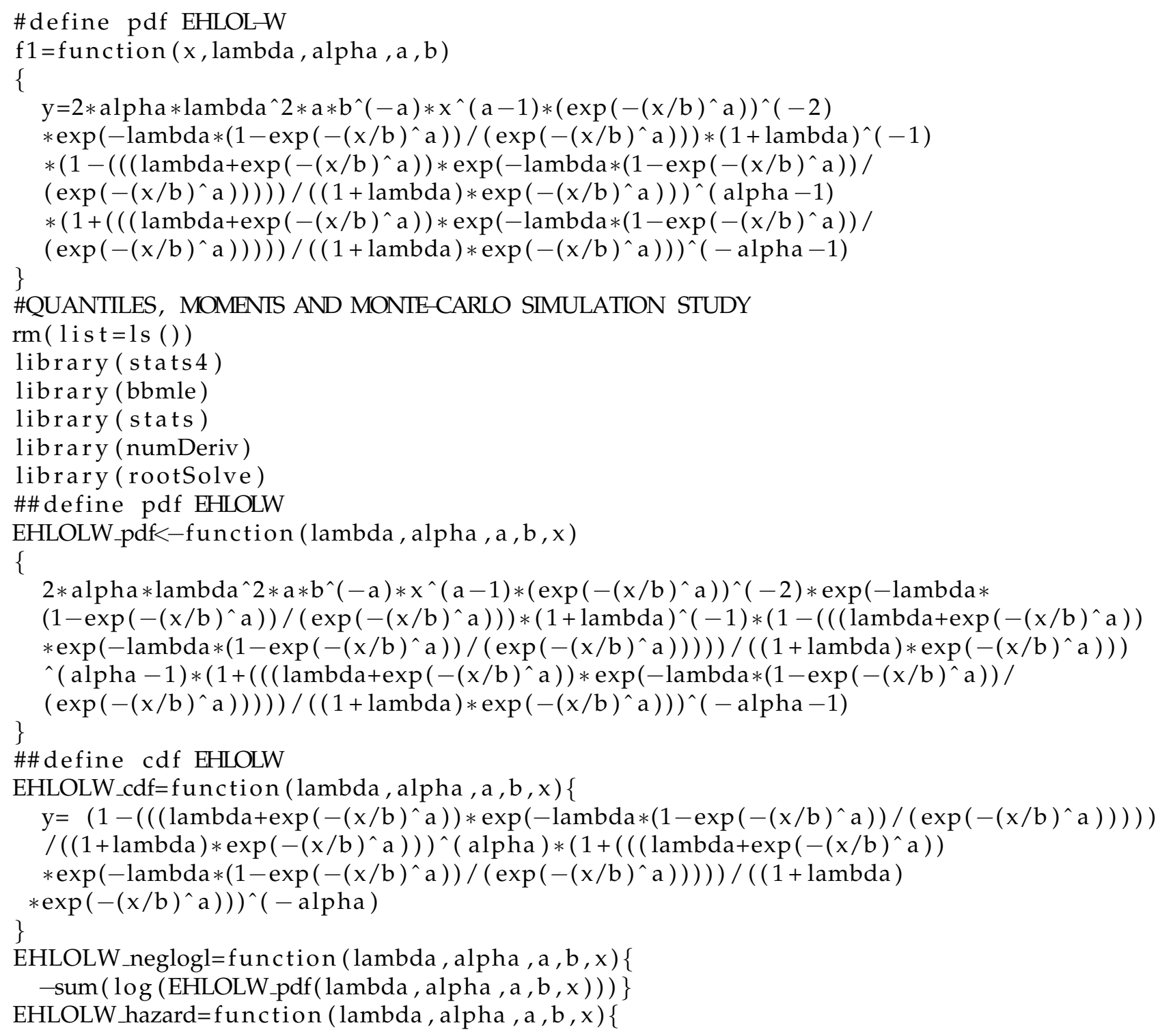




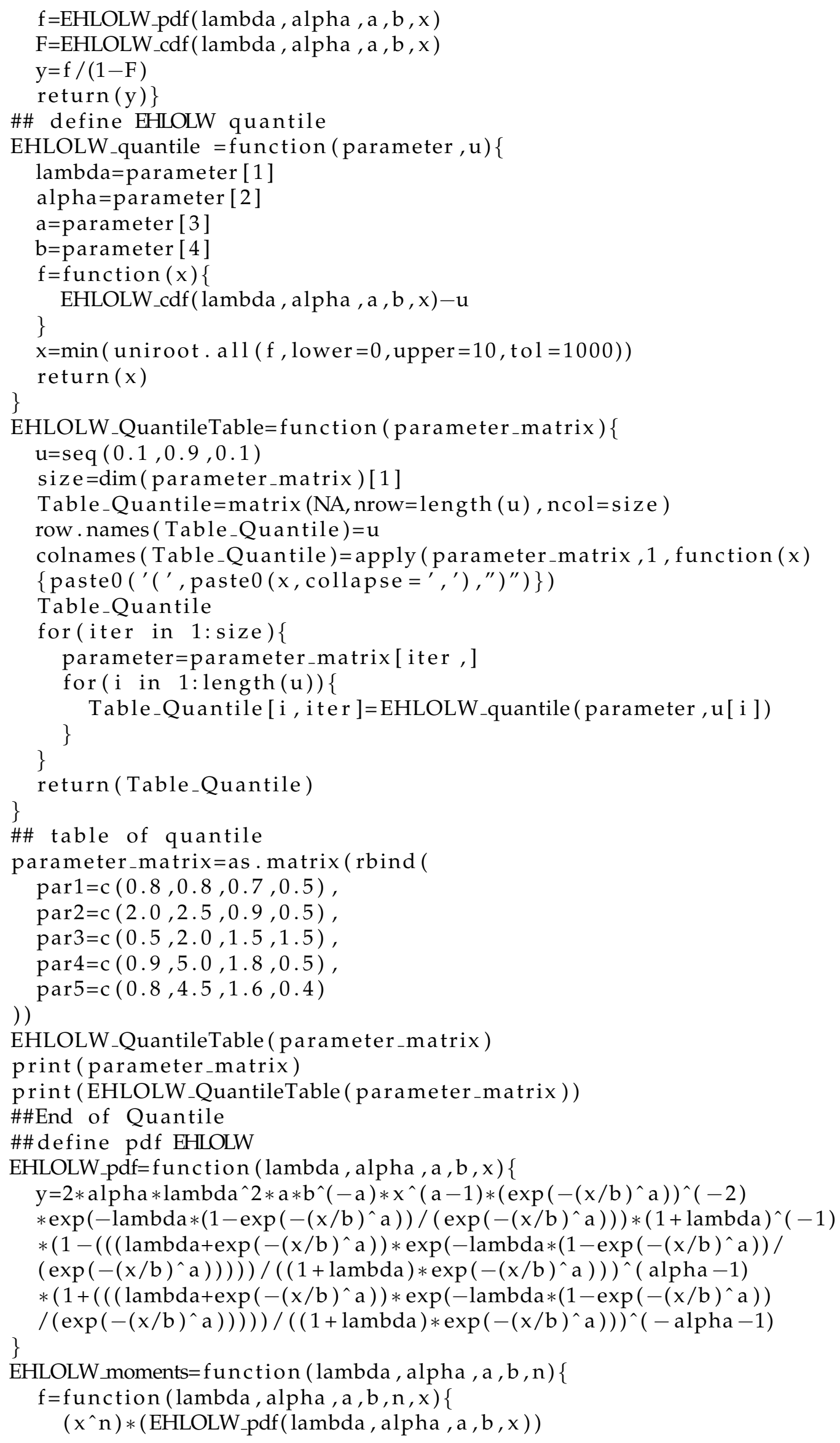




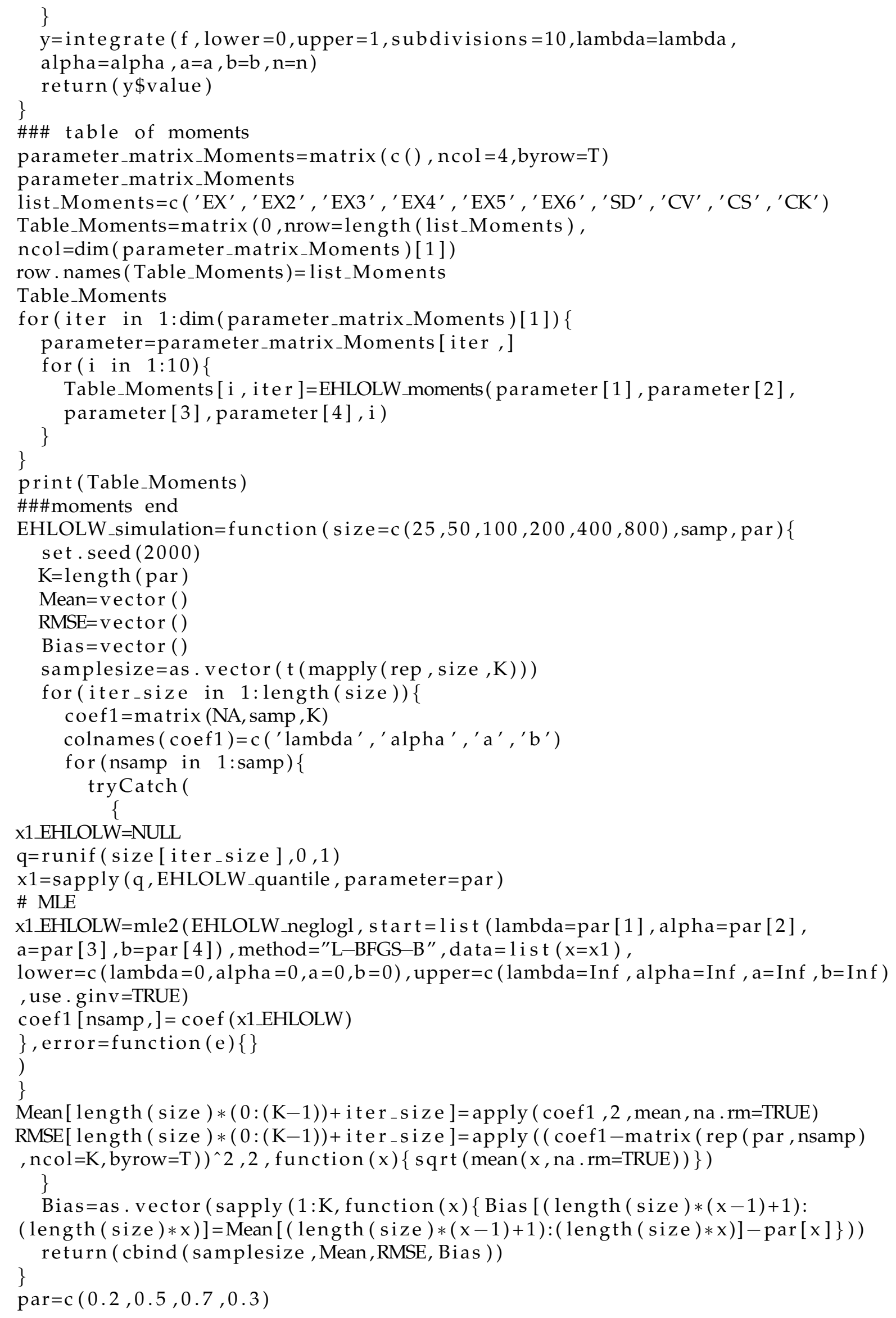




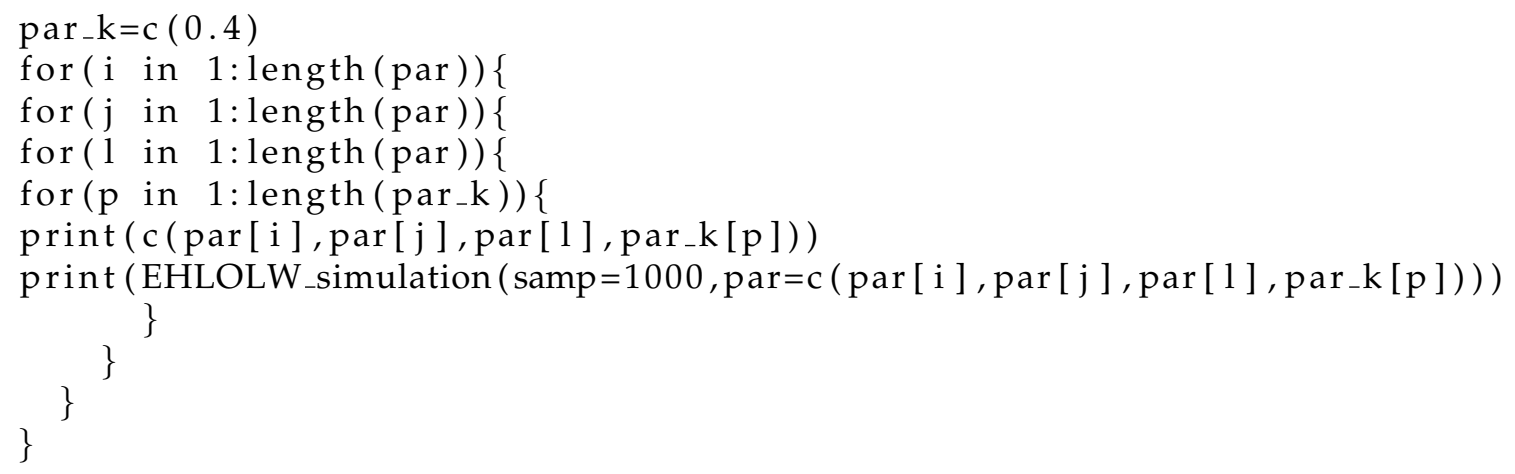

